\title{
The effect of tangential drifts on neoclassical transport in stellarators close to omnigeneity
}

\author{
Iván Calvo ${ }^{1}$ \\ E-mail: ivan.calvo@ciemat.es \\ Felix I Parra ${ }^{2,3}$ \\ E-mail: felix.parradiaz@physics.ox.ac.uk \\ José Luis Velasco ${ }^{1}$ \\ E-mail: joseluis.velasco@ciemat.es \\ J Arturo Alonso ${ }^{1}$ \\ E-mail: arturo.alonso@ciemat.es \\ ${ }^{1}$ Laboratorio Nacional de Fusión, CIEMAT, 28040 Madrid, Spain \\ ${ }^{2}$ Rudolf Peierls Centre for Theoretical Physics, University of Oxford, Oxford, OX1 \\ $3 \mathrm{NP}, \mathrm{UK}$ \\ ${ }^{3}$ Culham Centre for Fusion Energy, Abingdon, OX14 3DB, UK \\ PACS numbers: 52.20.Dq, 52.25.Fi, 52.25.Xz, 52.55.Hc
}

\begin{abstract}
In general, the orbit-averaged radial magnetic drift of trapped particles in stellarators is non-zero due to the three-dimensional nature of the magnetic field. Stellarators in which the orbit-averaged radial magnetic drift vanishes are called omnigeneous, and they exhibit neoclassical transport levels comparable to those of axisymmetric tokamaks. However, the effect of deviations from omnigeneity cannot be neglected in practice, and it is more deleterious at small collisionalities. For sufficiently low collision frequencies (below the values that define the $1 / \nu$ regime), the components of the drifts tangential to the flux surface become relevant. This article focuses on the study of such collisionality regimes in stellarators close to omnigeneity when the gradient of the non-omnigeneous perturbation is small. First, it is proven that closeness to omnigeneity is required to actually preserve radial locality in the drift-kinetic equation for collisionalities below the $1 / \nu$ regime. Then, using the derived radially local equation, it is shown that neoclassical transport is determined by two layers located at different regions of phase space. One of the layers corresponds to the so-called $\sqrt{\nu}$ regime and the other to the so-called superbanana-plateau regime. The importance of the superbanana-plateau layer for the calculation of the tangential electric field is emphasized, as well as the relevance of the latter for neoclassical transport in the collisionality regimes considered in this paper. In particular, the role of the tangential electric field is essential for the emergence of a new subregime of superbanana-plateau transport when the radial electric field is small. A formula for the ion energy flux that includes the $\sqrt{\nu}$ regime and the superbanana-plateau regime is given. The energy flux scales with the square of the size of the deviation from omnigeneity. Finally, it is explained why below a certain collisionality value the formulation presented in this article ceases to be valid.
\end{abstract}




\section{Introduction}

Stellarators [1] offer some intrinsic advantages compared to tokamaks, such as the possibility of steady-state operation and the absence of disruptions. However, the magnetic configuration of a stellarator has to be designed very carefully for it to have confinement properties comparable to those of an axisymmetric tokamak. In a generic stellarator, trapped particle orbits have non-zero secular radial drifts and they leave the device in a short time. The stellarator configuration is called omnigeneous $[2,3,4,5]$ if the secular radial drifts vanish.

Omnigeneity guarantees a neoclassical transport level similar to that in a tokamak (see equation (2) below). Define the normalized ion gyroradius $\rho_{i *}:=v_{t i} /\left(\Omega_{i} L_{0}\right)$, where $v_{t i}$ and $\Omega_{i}$ are the ion thermal speed and the ion gyrofrequency, and $L_{0}$ is the typical length of variation of the magnetic field, which is assumed to be of the order of the system size. The gyrofrequency is $\Omega_{i}=Z_{i} e B /\left(m_{i} c\right)$, where $Z_{i} e$ is the charge of the ions, $e$ is the elementary charge, $B$ is the magnitude of the magnetic field $\mathbf{B}, m_{i}$ is the ion mass, and $c$ is the speed of light. Since $\rho_{i *} \ll 1$ in a strongly magnetized plasma, the driftkinetic formalism [6] is appropriate. Denoting by $f_{i}(\mathbf{r}, \mathbf{v})$ the phase-space distribution, the radial ion energy flux $Q_{i}$ reads

$$
Q_{i}=\int \mathrm{d}^{2} S \int \mathrm{d}^{3} v\left(\frac{m_{i} v^{2}}{2}+Z_{i} e \varphi\right) \mathbf{v}_{d, i} \cdot \hat{\mathbf{n}} f_{i} .
$$

Here, $\varphi$ is the electrostatic potential, $\mathbf{v}_{d, i}$ is the drift velocity, $\hat{\mathbf{n}}$ is the unit vector normal to the flux surface, $\mathbf{v}_{d, i} \cdot \hat{\mathbf{n}} \sim \rho_{i *} v_{t i}$, and the integrals are performed over velocity space and over the flux surface. In a perfectly omnigeneous stellarator $f_{i}$, to lowest order in $\rho_{i *}$, is a Maxwellian $f_{M i}$ with density $n_{i}$ and temperature $T_{i}$ that are constant on flux surfaces. The phase-space distribution is written as $f_{i}=f_{M i}+f_{i 1}$, where the perturbation to the Maxwellian is found to have a size $f_{i 1} \sim O\left(\rho_{i *} f_{M i}\right)$. The first nonvanishing contribution to the energy flux comes from a piece of the distribution function that is $O\left(\nu_{i *} \rho_{i *} f_{M i}\right)$, where $\nu_{i *}:=\nu_{i i} L_{0} / v_{t i}$ is the ion collisionality and $\nu_{i i}$ is the ion-ion collision frequency. Then, in an omnigeneous stellarator,

$$
Q_{i} \sim \nu_{i *} \rho_{i *}^{2} n_{i} T_{i} v_{t i} S_{\psi} .
$$

The area of the flux surface is denoted by $S_{\psi}$, with $\psi$ the radial coordinate.

The proof of Cary and Shasharina $[2,3]$ for the existence of omnigeneous magnetic fields implies that exact omnigeneity throughout the plasma requires, at least, nonanaliticity. Let us explain this in more detail. As shown in references [2] and [3], there exist omnigeneous magnetic fields that are analytic. These configurations coincide with the set of quasisymmetric magnetic fields $[7,8]$. To the virtues of omnigeneity, quasisymmetry adds the vanishing of neoclassical flow damping in the quasisymmetric direction. Therefore, in quasisymmetric stellarators larger flow velocities can be attained. In principle, a quasisymmetric stellarator plasma may have large flow shear, that in principle can reduce turbulent transport [9]. However, the quasisymmetry condition is incompatible with the magnetohydrodynamic equilibrium equations [10], and the stellarator can be made quasisymmetric only in a limited radial region. 
This is why we said above that a necessary condition for exact omnigeneity is nonanaliticity; specifically, the discontinuity of some derivatives of second or higher order. However, designing and aligning coils that create a magnetic field with discontinuous derivatives at certain points in space is probably technically impossible. Therefore, even in optimized magnetic fields, the effect of deviations from the desired omnigeneous configuration cannot be neglected. It is thus necessary to study magnetic fields of the form $\mathbf{B}=\mathbf{B}_{0}+\delta \mathbf{B}_{1}$, where $\mathbf{B}_{0}$ is omnigeneous and $\delta \mathbf{B}_{1}$ is a perturbation, with $0 \leq \delta \ll 1$ and $\mathbf{B}_{1} \sim \mathbf{B}_{0}$.

The effect of a deviation from omnigeneity is more detrimental for confinement at small collisionalities. If $\nu_{i *} \ll 1$ and the stellarator is non-omnigeneous, the nonomnigeneous piece of $f_{i 1}$ becomes large, so that $f_{i 1} \gg \rho_{i *} f_{M i}$ and the energy flux can be much larger than the estimation (2) even if $\delta$ is small. The quantification of this effect for

$$
\rho_{i *} \ll \nu_{i *} \ll 1,
$$

that defines the $1 / \nu$ regime, has been treated in $[11,12,13]$ for stellarators close to quasisymmetry and is the subject of [14] for stellarators close to omnigeneity. However, this regime does not exhaust the low collisionality parameter space in stellarators. When

$$
\nu_{i *} \lesssim \rho_{i *}
$$

the components of the drifts tangential to the flux surface matter $[1,15,16,17]$. In this paper we study stellarators close to omnigeneity in the collisionality regime (4), relevant for a stellarator reactor [18].

It is important to point out that the calculations in this paper do not rely on large aspect ratio approximations. Of course, if the stellarator close to omnigeneity under consideration has large aspect ratio, one can perform a subsidiary expansion in the inverse aspect ratio and refine the results obtained here. This will be the subject of future work. The rest of the paper is organized as follows.

In Section 2 we introduce a set of flux coordinates that is well-adapted to stellarator magnetic geometries. Then, we give the formal definition of omnigeneity.

In Section 3 we derive, starting from the complete drift-kinetic equation, the equation for the dominant component of the distribution function when $\delta \ll 1$ and $\nu_{i *} \lesssim \rho_{i *}$. In particular, we explain why the standard expansion in $\rho_{i *}$ breaks down for a generic stellarator when $\nu_{i *} \lesssim \rho_{i *}$. In brief, the reason is that $f_{i 1}$ becomes so large that $f_{i 1} \sim f_{M i}$. For stellarators close to omnigeneity, however, we can expand in the small parameter $\delta$. In addition, in a generic stellarator the drift-kinetic equation becomes radially non-local when $\nu_{i *} \lesssim \rho_{i *}$, but we will see that with the condition $\delta \ll 1$ we can derive a radially local drift-kinetic equation in this collisionality regime.

A precision must be made about the asymptotic expansion in $\delta$ carried out in this paper. When $\rho_{i *} \ll \nu_{i *} \ll 1$, it has been understood (in $[11,12,13]$ for stellarators close to quasisymmetry and in [14] for stellarators close to omnigeneity) that the effect of the deviations (from quasisymmetry or omnigeneity) is very different depending on the size of the gradients on the surface of the magnetic field perturbation. For the regime 
(4), the case of deviations with small gradients and the case of deviations with large gradients also require different treatments, in principle. Here, we restrict ourselves to deviations with small gradients. Let us be more precise. If $B_{0}:=\left|\mathbf{B}_{0}\right|$ and $B_{1}:=\left|\mathbf{B}_{1}\right|$, by "deviations with small gradients" we mean that $\left|\nabla \ln B_{0}\right| /\left|\nabla \ln B_{1}\right| \gg \delta$. If this inequality is well satisfied, then we can consider that the characteristic lengths of both, $B_{0}$ and $B_{1}$, are $O\left(L_{0}\right)$ as far as the asymptotic expansion in $\delta$ is concerned.

In Section 4 the equation derived in Section 3 for the non-omnigeneous piece of the distribution function is solved when $\nu_{i *} \ll \rho_{i *}$. We find that $Q_{i}$ is dominated by two collisional layers in phase space. One of the layers lies at the boundary between trapped and passing trajectories and produces an energy flux

$$
Q_{i} \sim \delta^{2} \frac{\nu_{i i}^{1 / 2}}{\omega_{\alpha}^{3 / 2}} \sqrt{\ln \left(\omega_{\alpha} / \nu_{i i}\right)} \rho_{i *}^{2} n_{i} T_{i} v_{t i}^{2} L_{0}^{-1} S_{\psi},
$$

where $\omega_{\alpha} \sim \rho_{i *} v_{t i} / L_{0}$, defined in Section 4 , is the precession frequency due to the tangential drifts. On the right side of (5) $\omega_{\alpha}$ actually stands for the value of the precession frequency evaluated at the boundary between trapped and passing particles and at $v=v_{t i}$. Note the logarithmic correction to the scaling with $\left(\nu_{i i} / \omega_{\alpha}\right)^{1 / 2}$ in $(5)$, that we calculate in subsection 4.1.

The other layer lies at the points of phase space where $\omega_{\alpha}$ vanishes and yields $Q_{i}$ independent of $\nu_{i *}$. Namely,

$$
Q_{i} \sim \delta^{2} \rho_{i *} n_{i} T_{i} v_{t i} S_{\psi}
$$

The first layer (see subsection 4.1 ) gives the so-called $\sqrt{\nu}$ regime, found in certain models of stellarator geometry [19, 20] where the inverse aspect ratio and the helical ripple are employed as expansion parameters. The second layer (see subsection 4.2) gives the superbanana-plateau regime, derived in [21] for finite aspect ratio tokamaks with broken symmetry. Here, the $\sqrt{\nu}$ and superbanana-plateau regimes are derived and analyzed in a much broader setting and in deeper detail than previously available in the literature. In particular, we will show that the treatment of the superbanana-plateau regime requires special care for small values of the radial electric field (see (150) for a precise definition of what 'small' means in this context), and in those cases logarithmic corrections appear in (6) as well. Of course, neither the $\sqrt{\nu}$ regime nor the superbanana-plateau regime (nor the $1 / \nu$ regime) exist in perfectly omnigeneous stellarators; i.e. when $\delta=0$.

From the start, it will be evident that the role of the electric field tangent to the flux surface is relevant when the collisionality is as low as in (4) (not to mention its importance for impurity transport, as pointed out, for example, in [22, 23, 24]). Furthermore, we will show that writing the contributions to the quasineutrality equation that gives the electric field tangent to the flux surface is a subtle issue. In particular, we will prove that the superbanana-plateau layer has to be resolved to find the tangent electric field. For this reason, we discuss the quasineutrality equation in subsection 4.2.1.

The contributions to $Q_{i}$ from the two layers mentioned above are additive, as long as the layers are distinct and do not overlap, and a general expression embracing the $\sqrt{\nu}$ 
and superbanana-plateau regimes is provided in subsection 4.3. The treatment of cases in which both layers overlap is left for the future. As (5) and (6) already indicate, we will show that the neoclassical fluxes scale with the square of the size of the deviation from omnigeneity, $\delta$.

In Section 5 we use the results of previous sections to write the equation that gives the radial electric field.

Finally, in Section 6, we explain that the results of Section 4 are not expected to be correct for arbitrarily small $\nu_{i *}$. For each $\delta$ there exists a value of the collisionality $\nu_{\delta *}$ such that if $\nu_{i *}<\nu_{\delta *}$ our solution is not valid. We explain and estimate the limit value $\nu_{\delta *}$.

In Section 7 we summarize the conclusions of the paper.

\section{Omnigeneous stellarators}

Throughout the paper, we deal with stellarators whose magnetic field configurations possess nested flux surfaces. In the first place, we define a set of spatial coordinates $\{\psi, \alpha, l\}$ adapted to the magnetic field. The coordinate $\psi$ determines the flux surface, whereas $\alpha$ is an angular coordinate that labels a magnetic field line once $\psi$ has been fixed. Finally $l$, the arc length over the magnetic field line, specifies the position along the line for fixed $\psi$ and $\alpha$. Denote by $\psi(\mathbf{r}), \alpha(\mathbf{r})$ and $l(\mathbf{r})$ the functions giving the value of these coordinates for each point $\mathbf{r}$ in the stellarator. The magnetic field can be written as

$$
\mathbf{B}=\Psi_{t}^{\prime}(\psi) \nabla \psi \times \nabla \alpha
$$

Here, $\Psi_{t}$ is the toroidal magnetic flux over $2 \pi$ and primes stand for differentiation with respect to $\psi$. In order to have unique pairs $(\alpha, l)$ associated to each point on a flux surface, we choose a curve $\mathcal{C}$ that closes poloidally $\ddagger$. This curve can be parameterized by $\alpha$. All points on the curve are assigned, by definition, the value $l=0$. For each pair $\psi$ and $\alpha$ we take $l \in[0, L(\psi, \alpha))$, where $L(\psi, \alpha)$ is found by integrating from $\mathcal{C}$ along the line until the curve $\mathcal{C}$ is encountered again.

Let $v$ be the magnitude of the velocity and $\lambda=v_{\perp}^{2} /\left(v^{2} B\right)$ the pitch angle. Given a flux surface determined by $\psi$, particles are passing or trapped depending on the value of $\lambda$. Passing trajectories have $\lambda<1 / B_{\max }(\psi)$, where $B_{\max }(\psi)$ is the maximum value of $B$ on the flux surface. Passing particles explore the entire flux surface and always have vanishing average radial magnetic drift. Particles with $\lambda>1 / B_{\max }(\psi)$ are trapped. For trapped particles, the radial magnetic drift averaged over the orbit is non-zero in a generic stellarator. A stellarator is called omnigeneous if the orbit-averaged radial magnetic drift is zero for all particles $[2,3,4,5]$. That is, if and only if the second

$\ddagger$ To fix ideas, we are thinking of $\alpha$ as a poloidal angle, but things work analogously if $\alpha$, and therefore the curve $\mathcal{C}$, have a different helicity. 
adiabatic invariant $J=2 \int_{l_{b_{1}}}^{l_{b_{2}}}\left|v_{\|}\right| \mathrm{d} l$ is a flux function, which means that

$$
\partial_{\alpha} \int_{l_{b_{1}}}^{l_{b_{2}}} \sqrt{1-\lambda B} \mathrm{~d} l=0
$$

must hold for every trapped trajectory. Here $l_{b_{1}}$ and $l_{b_{2}}$ are the bounce points; i.e. the solutions for $l$ of the equation $1-\lambda B(\psi, \alpha, l)=0$ for a particular trapped trajectory. Since (8) has to be satisfied for every $\lambda$, that equation is equivalent to requiring $\S$

$$
\partial_{\alpha} \int_{l_{b_{1}}}^{l_{b_{2}}} \Lambda(\psi, v, \lambda, B(\psi, \alpha, l)) \mathrm{d} l=0
$$

for any function $\Lambda$ that depends on $\alpha$ and $l$ only through $B$. We will make use of this definition of omnigeneity several times along the article.

\section{Low-collisionality drift-kinetic equation in stellarators close to omnigeneity}

As we said in the Introduction, due to the smallness of $\rho_{i *}$ we can employ the drift-kinetic approach $[6,11,25,26]$. It consists of a systematic way to average, order by order in $\rho_{i *}$, over the fast gyration of particles around magnetic field lines. This is achieved by finding a coordinate transformation on phase space that decouples the gyromotion from the comparatively slow motion of the guiding center. The new coordinates are called drift-kinetic coordinates. In what follows, we restrict ourselves to electrostatic drift-kinetics and assume that $\varphi \sim m_{i} v_{t i}^{2} / e$.

The form of the drift-kinetic equation is determined by the transformation from coordinates $\{\mathbf{r}, \mathbf{v}\}$ to drift-kinetic coordinates (or, perhaps more precisely, to the driftkinetic limit of gyrokinetic coordinates [27]). Even though we will end up employing the coordinates $v$ and $\lambda$ defined in Section 2, it is convenient to start using as independent coordinates the total energy per mass unit $\mathcal{E}$ and the magnetic moment $\mu$ because they are constants of the particle motion. Then, in drift-kinetic coordinates $\{\mathbf{R}, \mathcal{E}, \mu, \sigma, \gamma\}$, where $\mathbf{R}$ is the position of the guiding center, $\sigma$ is the sign of the parallel velocity and $\gamma$ is the gyrophase, we have

$$
\begin{aligned}
& \mathbf{R}=\mathbf{r}-\frac{1}{\Omega_{i}} \hat{\mathbf{b}} \times \mathbf{v}+O\left(\rho_{i}^{* 2} L_{0}\right), \\
& \mathcal{E}=\frac{v^{2}}{2}+\frac{Z_{i} e \varphi}{m_{i}} \\
& \mu=\frac{1}{2 B}\left(v^{2}-(\mathbf{v} \cdot \hat{\mathbf{b}})^{2}\right)+O\left(\rho_{i *} v_{t i}^{2} / B\right), \\
& \gamma=\arctan \left(\frac{\mathbf{v} \cdot \hat{\mathbf{e}}_{2}}{\mathbf{v} \cdot \hat{\mathbf{e}}_{1}}\right)+O\left(\rho_{i *}\right),
\end{aligned}
$$

$\S$ In [3], it is proven that (8) implies that $\sum|\mathrm{d} l / \mathrm{d} B|$ (the sum runs over the two points of each well where the magnitude of the magnetic field reaches a certain value $B$ ) depends only on $\psi$ and on the value of $B$. Property (9) follows by employing this result after changing the integration variable on the left-side of (9) from $l$ to $B$. 
where $\hat{\mathbf{b}}=B^{-1} \mathbf{B}$ and the right sides of the previous expressions are evaluated at $\mathbf{r}$. The orthogonal unit vector fields $\hat{\mathbf{e}}_{1}$ and $\hat{\mathbf{e}}_{2}$ satisfy at each point $\hat{\mathbf{e}}_{1} \times \hat{\mathbf{e}}_{2}=\hat{\mathbf{b}}$. The higher-order corrections in the definition of $\mu$ are determined by the fact that $\mu$ is the adiabatic invariant corresponding to the ignorable coordinate $\gamma$. Finally, $\sigma=v_{\|} /\left|v_{\|}\right|$ gives the sign of the parallel velocity, where the latter is viewed as a function of the other coordinates through the expression

$$
v_{\|}=\sigma \sqrt{2\left(\mathcal{E}-\mu B-\frac{Z_{i} e \varphi}{m_{i}}\right)} .
$$

Denote by $F_{i}(\psi(\mathbf{R}), \alpha(\mathbf{R}), l(\mathbf{R}), \mathcal{E}, \mu, \sigma)$ the distribution function in drift-kinetic coordinates. We assume from the beginning that our distribution function does not depend on the gyrophase $\gamma$, which is true for all the calculations in this paper (see [25] for the proof that only pieces of the distribution function $O\left(\nu_{i *} \rho_{i *} f_{M i}\right)$ or smaller are gyrophase dependent). In these coordinates the drift-kinetic equation reads

$$
\dot{\mathbf{R}} \cdot \nabla F_{i}=C_{i i}^{\mathcal{E}}\left[F_{i}, F_{i}\right] .
$$

Here,

$$
\dot{\mathbf{R}} \cdot \hat{\mathbf{b}} \hat{\mathbf{b}}=v_{\|} \hat{\mathbf{b}}+O\left(\rho_{i *} v_{t i}\right)
$$

and

$$
\dot{\mathbf{R}}-\dot{\mathbf{R}} \cdot \hat{\mathbf{b}} \hat{\mathbf{b}}=\mathbf{v}_{M, i}+\mathbf{v}_{E}+O\left(\rho_{i}^{* 2} v_{t i}\right),
$$

with

$\mathbf{v}_{M, i}=\frac{1}{\Omega_{i}} \hat{\mathbf{b}} \times\left(v_{\|}^{2} \boldsymbol{\kappa}+\mu \nabla B\right)$

being the magnetic drift,

$\mathbf{v}_{E}=\frac{c}{B} \hat{\mathbf{b}} \times \nabla \varphi$

being the $E \times B$ drift and $\boldsymbol{\kappa}=\hat{\mathbf{b}} \cdot \nabla \hat{\mathbf{b}}$ being the curvature of the magnetic field lines. Note that $\left|\mathbf{v}_{M, i}\right|$ and $\left|\mathbf{v}_{E}\right|$ are $O\left(\rho_{i *} v_{t i}\right)$.

In (13) and (14) we have shown only the terms that will be needed later on. All the terms of $\dot{\mathbf{R}}$ up to $O\left(\rho_{i}^{* 2} v_{t i}\right)$ have been computed in [26]. In (12), an expansion in the mass ratio $\sqrt{m_{e} / m_{i}} \ll 1$ has been taken so that ion-electron collisions are neglected, and $C_{i i}^{\mathcal{E}}$ is the ion-ion Landau collision operator written in coordinates $\mathcal{E}$ and $\mu$. Its explicit expression (see [28], for example) is not necessary for our purposes. From here on, we concentrate on ion transport.

Low collisionality regimes are defined by $\nu_{i *} \ll 1$. It is well-known (see, for example, subsection 7.1 in [11] and also [14]) that if the collisionality is small but still larger than the normalized gyroradius, i.e. if $\rho_{i *} \ll \nu_{i *} \ll 1$, then the distribution function and electrostatic potential can be expanded as

$$
F_{i}=F_{i 0}^{\mathcal{E}}+F_{i 1}+\ldots
$$

and

$$
\varphi=\varphi_{0}+\varphi_{1}+\ldots
$$


where

$$
F_{i 0}^{\mathcal{E}}(\psi, \mathcal{E})=n_{i}(\psi)\left(\frac{m_{i}}{2 \pi T_{i}(\psi)}\right)^{3 / 2} \exp \left(-\frac{m_{i} \mathcal{E}-Z_{i} e \varphi_{0}(\psi)}{T_{i}(\psi)}\right)
$$

is a Maxwellian distribution with density and temperature constant on the flux surface, the non-adiabatic perturbation to $F_{i 0}^{\mathcal{E}}$ has a size

$$
F_{i 1} \sim \frac{\rho_{i *}}{\nu_{i *}} F_{i 0}^{\mathcal{E}},
$$

$\varphi_{0}(\psi) \sim T_{i} / Z_{i}$ e is a flux function and $\varphi_{1}$ is found from the quasineutrality equation

$$
Z_{i} \int F_{i} \mathrm{~d}^{3} v=N_{e}
$$

Here, $N_{e}$ is the electron density and $\int(\cdot) \mathrm{d}^{3} v \equiv \sum_{\sigma} \int(\cdot) B\left|v_{\|}\right|^{-1} \mathrm{~d} \mathcal{E} \mathrm{d} \mu \mathrm{d} \gamma$. To lowest order in $\sqrt{m_{e} / m_{i}} \ll 1$, only the adiabatic response of the electrons counts. Then,

$$
N_{e}=n_{e}(\psi)\left\langle\exp \left(\frac{e \varphi}{T_{e}(\psi)}\right)\right\rangle_{\psi}^{-1} \exp \left(\frac{e \varphi}{T_{e}(\psi)}\right),
$$

where $T_{e}$ is the electron temperature, $n_{e}$ is the flux-surface averaged electron density and $\langle\cdot\rangle_{\psi}$ denotes the flux-surface average operation, defined for a function $f(\psi, \alpha, l)$ as

$$
\langle f\rangle_{\psi}=V^{\prime}(\psi)^{-1} \int_{0}^{2 \pi} \mathrm{d} \alpha \int_{0}^{L(\psi, \alpha)} \mathrm{d} l \Psi_{t}^{\prime} B^{-1} f,
$$

where $V^{\prime}(\psi)$ is the radial derivative of the volume enclosed by the flux surface labeled by $\psi$,

$$
V^{\prime}(\psi)=\int_{0}^{2 \pi} \mathrm{d} \alpha \int_{0}^{L(\psi, \alpha)} \mathrm{d} l \Psi_{t}^{\prime} B^{-1} .
$$

In the quasineutrality equation defined by (21) and (22) the expansions (17) and (18) have not been employed yet. Using them, we obtain

$$
\left(\frac{Z_{i}}{T_{i}}+\frac{1}{T_{e}}\right) \varphi_{1}=\frac{1}{e n_{i}} \int F_{i 1} \mathrm{~d}^{3} v
$$

where we have assumed that $\varphi_{1}$ and the right side of (25) have vanishing flux-surface average. The proof that this choice in the definition of $\varphi_{1}$ and $F_{i 1}$ is possible is provided in reference [11].

From (25) and the fact that $F_{i 1} \sim \nu_{i *}^{-1} \rho_{i *} F_{i 0}^{\mathcal{E}}$, one obtains

$$
\varphi_{1} \sim \frac{\rho_{i *}}{\nu_{i *}} \varphi_{0}
$$

This is the so-called $1 / \nu$ regime [29], that exists for any stellarator (strictly speaking, for any stellarator that is not exactly omnigeneous).

The point that needs to be emphasized here is that the expansions (17) and (18) do not work when $\nu_{i *} \lesssim \rho_{i *}$ because $F_{i 1}$ becomes as large as $F_{i 0}^{\mathcal{E}}$ and $\varphi_{1}$ becomes as large as $\varphi_{0}$ (see (20) and (26)). The regime $\nu_{i *} \lesssim \rho_{i *}$ is the subject of this paper, and we start to analyze it in the next subsection. 


\subsection{Drift-kinetic equation when $\nu_{i *} \lesssim \rho_{i *}$ in stellarators close to omnigeneity}

As explained above, the expansion of the distribution function and electrostatic potential employed in the $1 / \nu$ regime (recall equations (17), (19), (20), (18) and (26)), $\rho_{i *} \ll \nu_{i *} \ll$ 1 , is not valid when $\nu_{i *} \lesssim \rho_{i *}$. In order to understand what happens at collisionality values $\nu_{i *} \lesssim \rho_{i *}$ we go back to (12), assume $\nu_{i *} \sim \rho_{i *}$ and expand in $\rho_{i *}$.

We take

$$
F_{i}=F_{i 0}^{\mathcal{E}}+F_{i 1}+\ldots
$$

with $F_{i 1} \sim \rho_{i *} F_{i 0}^{\mathcal{E}}$. To lowest order in $\rho_{i *}$ equation (12) gives

$$
v_{\|} \partial_{l} F_{i 0}^{\mathcal{E}}=0 .
$$

To solve (28) and the next order equations, we employ a procedure similar to the one developed in [14] for the $1 / \nu$ regime. Equation (28) implies that on an ergodic flux surface $\| F_{i 0}^{\mathcal{E}}$ can be written as

$$
F_{i 0}^{\mathcal{E}}=h_{i}(\psi, \mathcal{E}, \mu, \sigma)+g_{i}(\psi, \alpha, \mathcal{E}, \mu),
$$

where $g_{i}$ can be chosen such that it vanishes in the passing particle region of phase space and $h_{i}$ cannot depend on $\sigma$ in the trapped particle region. In order to understand (29) observe, first, that the distribution function $F_{i 0}^{\mathcal{E}}$ cannot depend on $\alpha$ in the passing region of phase space because passing particles trace out a flux surface. Second, that in the trapped region of phase space $F_{i 0}^{\mathcal{E}}$ cannot depend on $\sigma$ because it has to be continuous at the bounce points. The split between $h_{i}$ and $g_{i}$ is defined up to a function independent of $\alpha$ that vanishes for passing particles. To completely determine $g_{i}$, we impose the condition

$$
\int_{0}^{2 \pi} \mathrm{d} \alpha \int_{l_{b_{1}}}^{l_{b_{2}}} \mathrm{~d} l \frac{g_{i}}{\left|v_{\mid}\right|}=0 .
$$

There are other conditions that could be used to fix $g_{i}$.

The equation satisfied by $F_{i 0}^{\mathcal{E}}$ is found from averages of equation (12) to next order in $\rho_{i *}$. For passing particles one has to multiply the $O\left(\rho_{i *} v_{t i} L_{0}^{-1} F_{i 0}^{\mathcal{E}}\right)$ terms of $(12)$ by $1 /\left|v_{\|}\right|$and integrate over the flux surface, obtaining

$\int_{0}^{2 \pi} \mathrm{d} \alpha \int_{0}^{L(\psi, \alpha)} \frac{1}{\left|v_{\|}\right|} C_{i i}^{\mathcal{E}}\left[F_{i 0}^{\mathcal{E}}, F_{i 0}^{\mathcal{E}}\right] \mathrm{d} l=0$.

In order to get $(31)$ we have employed $\partial_{l} F_{i 0}^{\mathcal{E}}=0$, the fact that in the passing region $\partial_{\alpha} F_{i 0}^{\mathcal{E}}=0$, and finally the property

$\int_{0}^{2 \pi} \mathrm{d} \alpha \int_{0}^{L(\psi, \alpha)} \frac{1}{\left|v_{\|}\right|}\left(\mathbf{v}_{M, i}+\mathbf{v}_{E}\right) \cdot \nabla \psi \mathrm{d} l=0$

for passing trajectories.

\| On a rational surface, passing particles follow periodic orbits and must be treated like standard trapped particles. Hence, there would be no splitting between $h_{i}$ and $g_{i}$ on a rational surface. 
For trapped particles we multiply the $O\left(\rho_{i *} v_{t i} L_{0}^{-1} F_{i 0}^{\mathcal{E}}\right)$ terms of (12) by $1 / v_{\|}$and integrate over the orbit, arriving at

$-\partial_{\psi} J \partial_{\alpha} F_{i 0}^{\mathcal{E}}+\partial_{\alpha} J \partial_{\psi} F_{i 0}^{\mathcal{E}}=\sum_{\sigma} \frac{Z_{i} e \Psi_{t}^{\prime}}{m_{i} c} \int_{l_{b_{1}}}^{l_{b_{2}}} \frac{1}{\left|v_{\|}\right|} C_{i i}^{\mathcal{E}}\left[F_{i 0}^{\mathcal{E}}, F_{i 0}^{\mathcal{E}}\right] \mathrm{d} l$.

Equation (33) has conveniently been expressed in terms of the second adiabatic invariant

$$
J(\psi, \alpha, \mathcal{E}, \mu):=2 \int_{l_{b_{1}}}^{l_{b_{2}}}\left|v_{\|}\right| \mathrm{d} l
$$

by employing the relations

$2 \int_{l_{b_{1}}}^{l_{b_{2}}} \frac{1}{\left|v_{\|}\right|}\left(\mathbf{v}_{M, i}+\mathbf{v}_{E}\right) \cdot \nabla \psi \mathrm{d} l=\frac{m_{i} c}{Z_{i} e \Psi_{t}^{\prime}} \partial_{\alpha} J$

and

$2 \int_{l_{b_{1}}}^{l_{b_{2}}} \frac{1}{\left|v_{\|}\right|}\left(\mathbf{v}_{M, i}+\mathbf{v}_{E}\right) \cdot \nabla \alpha \mathrm{d} l=-\frac{m_{i} c}{Z_{i} e \Psi_{t}^{\prime}} \partial_{\psi} J$

that are derived in Appendix A.

Given the profiles for ion density, ion temperature and radial electric field, the piece of the electrostatic potential that determines the tangential electric field is found from (recall (21) and (22))

$$
Z_{i} \int F_{i 0}^{\mathcal{E}} \mathrm{d}^{3} v=n_{e}(\psi)\left\langle\exp \left(\frac{e \varphi}{T_{e}(\psi)}\right)\right\rangle_{\psi}^{-1} \exp \left(\frac{e \varphi}{T_{e}(\psi)}\right) .
$$

In a generic stellarator one cannot go beyond (31), (33) and (37), that are a set of non-linear equations for the distribution function and the electrostatic potential. In particular, this means that without further assumptions, when $\nu_{i *} \lesssim \rho_{i *}$, one cannot deduce that $F_{i 0}^{\mathcal{E}}$ be Maxwellian, and the drift-kinetic equation is clearly not radially local (note the term in $(33)$ containing $\partial_{\psi} F_{i 0}^{\mathcal{E}}$ ). However, we proceed to show that the situation is different if the stellarator is close to omnigeneity.

We take

$$
\mathbf{B}=\mathbf{B}_{0}+\delta \mathbf{B}_{1}
$$

where $\mathbf{B}_{0}$ is omnigeneous, $\mathbf{B}_{0} \sim \mathbf{B}_{1}$ and $0 \leq \delta \ll 1$, and assume that the expansion in $\delta$ is subsidiary with respect to the expansion in $\rho_{i *}$. As advanced in the Introduction, we only consider the case in which $B_{1}$ has small spatial derivatives tangent to the flux surface,

$$
\begin{aligned}
& \delta \partial_{l} B_{1} \sim \delta L_{0}^{-1} B_{0}, \\
& \delta \partial_{\alpha} B_{1} \sim \delta B_{0} .
\end{aligned}
$$

The distribution function and the electrostatic potential are expanded as

$$
F_{i 0}^{\mathcal{E}}=F_{i 0}^{\mathcal{E}(0)}+\delta F_{i 0}^{\mathcal{E}(1)}+\ldots
$$

and

$$
\varphi=\varphi_{0}(\psi)+\delta \varphi^{(1)}+\ldots,
$$


where $F_{i 0}^{\mathcal{E}(1)} \sim F_{i 0}^{\mathcal{E}(0)}$ is the non-adiabatic correction of the distribution function and $\varphi^{(1)} \sim \varphi_{0}$. We also expand $J$ as

$$
J=J^{(0)}+\delta J^{(1)}+\ldots,
$$

with

$$
\begin{aligned}
& J^{(0)}=2 \int_{l_{b_{10}}}^{l_{b_{20}}}\left|v_{\|}^{(0)}\right| \mathrm{d} l, \\
& J^{(1)}=-2 \int_{l_{b_{10}}}^{l_{b_{20}}} \frac{1}{\left|v_{\|}^{(0)}\right|}\left(\mu B_{1}(\alpha, l)+\frac{Z_{i} e}{m_{i}} \varphi^{(1)}(\alpha, l)\right) \mathrm{d} l .
\end{aligned}
$$

Here $l_{b_{10}}$ and $l_{b_{20}}$ are the points that make

$v_{\|}^{(0)}(\psi, \alpha, l, \mathcal{E}, \mu)=\sigma \sqrt{2\left(\mathcal{E}-\mu B_{0}(\psi, \alpha, l)-\frac{Z_{i} e}{m_{i}} \varphi_{0}(\psi)\right)}$

vanish and $J^{(0)}$ is independent of $\alpha$, which is the defining property of omnigeneity, as explained in Section 2. The rigorous proof that the perturbation to $J^{(0)}$ is actually linear in $\delta$ when (39) is satisfied is contained in [30]. Finally, observe that we have assumed that $\varphi_{0}$ is a flux function. It can be proven that this follows from quasineutrality for an exactly omnigeneous magnetic field.

To lowest order in $\delta$ equation (31) gives

$\int_{0}^{2 \pi} \mathrm{d} \alpha \int_{0}^{L^{(0)}(\psi)} \frac{1}{\left|v_{\|}^{(0)}\right|} C_{i i}^{\mathcal{E}(0)}\left[F_{i 0}^{\mathcal{E}(0)}, F_{i 0}^{\mathcal{E}(0)}\right] \mathrm{d} l=0$,

where the superindex $(0)$ in $C_{i i}^{\mathcal{E}(0)}$ indicates that only $B_{0}$ has been kept in the kernel that defines the collision operator. Analogously, $L^{(0)}(\psi)$ is the length of the magnetic field line for the omnigeneous configuration, and it has been stressed that it does not depend on $\alpha$.

The lowest order terms of (33) in the $\delta$ expansion are

$-\partial_{\psi} J^{(0)} \partial_{\alpha} F_{i 0}^{\mathcal{E}(0)}=\sum_{\sigma} \frac{Z_{i} e \Psi_{t}^{\prime}}{m_{i} c} \int_{l_{b_{10}}}^{l_{b_{20}}} \frac{1}{\left|v_{\|}^{(0)}\right|} C_{i i}^{\mathcal{E}(0)}\left[F_{i 0}^{\mathcal{E}(0)}, F_{i 0}^{\mathcal{E}(0)}\right] \mathrm{d} l$,

where we have used $\partial_{\alpha} J^{(0)}=0$ due to omnigeneity.

We solve equations (45) and (46) by using the entropy production property of the collision operator. The lowest-order piece of $(29)$ in the $\delta$ expansion implies that $F_{i 0}^{\mathcal{E}(0)}$ does not depend on $\alpha$ in the passing region. Hence, we multiply (45) by $-\ln F_{i 0}^{\mathcal{E}(0)}$ and find

$-\int_{0}^{2 \pi} \mathrm{d} \alpha \int_{0}^{L^{(0)}(\psi)} \frac{1}{\left|v_{\|}^{(0)}\right|} \ln F_{i 0}^{\mathcal{E}(0)} C_{i i}^{\mathcal{E}(0)}\left[F_{i 0}^{\mathcal{E}(0)}, F_{i 0}^{\mathcal{E}(0)}\right] \mathrm{d} l=0$

in the passing region.

Similarly, we multiply (46) by $-\ln F_{i 0}^{\mathcal{E}(0)}$, integrate the resulting expression over $\alpha$ and recall that omnigeneity implies that $\partial_{\psi} J^{(0)}$ does not depend on $\alpha$. We end up with

$-\sum_{\sigma} \int_{0}^{2 \pi} \mathrm{d} \alpha \int_{l_{b_{10}}}^{l_{b_{20}}} \frac{1}{\left|v_{\|}^{(0)}\right|} \ln F_{i 0}^{\mathcal{E}(0)} C_{i i}^{\mathcal{E}(0)}\left[F_{i 0}^{\mathcal{E}(0)}, F_{i 0}^{\mathcal{E}(0)}\right] \mathrm{d} l=0$ 
in the trapped region. Integrating (47) and (48) in velocity space and following an entropy-production argument, we deduce that $F_{i 0}^{\mathcal{E}(0)}$ is a Maxwellian distribution. Furthermore, it must have zero flow because $F_{i 0}^{\mathcal{E}(0)}$ cannot depend either on $l$ or on the gyrophase. Inserting the Maxwellian into (46), we find that it is also independent of $\alpha$, leading to

$$
F_{i 0}^{\mathcal{E}(0)}(\psi, \mathcal{E})=n_{i}(\psi)\left(\frac{m_{i}}{2 \pi T_{i}(\psi)}\right)^{3 / 2} \exp \left(-\frac{m_{i} \mathcal{E}-Z_{i} e \varphi_{0}(\psi)}{T_{i}(\psi)}\right) .
$$

We turn to the equations provided by terms that are linear in $\delta$ in (31) and (33). Using the decomposition (29), we can write

$$
F_{i 0}^{\mathcal{E}(1)}=h_{i}^{(1)}(\psi, \mathcal{E}, \mu, \sigma)+g_{i}^{(1)}(\psi, \alpha, \mathcal{E}, \mu),
$$

where $h_{i}^{(1)}$ cannot depend on $\sigma$ in the trapped particle region of phase space, and $g_{i}^{(1)}$ may be chosen such that it vanishes in the passing particle region and such that

$$
\int_{0}^{2 \pi} g_{i}^{(1)} \mathrm{d} \alpha=0 .
$$

Equation (51) is simply condition (30) written to $O(\delta)$ by using (9).

To $O(\delta)$ equation $(31)$ gives

$\int_{0}^{2 \pi} \mathrm{d} \alpha \int_{0}^{L^{(0)}(\psi)} \frac{1}{\left|v_{\|}^{(0)}\right|} C_{i i}^{\mathcal{E}, \ell(0)}\left[h_{i}^{(1)}\right] \mathrm{d} l=0$,

where

$$
C_{i i}^{\mathcal{E}, \ell(0)}\left[h_{i}^{(1)}\right]=C_{i i}^{\mathcal{E}(0)}\left[F_{i 0}^{\mathcal{E}(0)}, h_{i}^{(1)}\right]+C_{i i}^{\mathcal{E}(0)}\left[h_{i}^{(1)}, F_{i 0}^{\mathcal{E}(0)}\right]
$$

is the linearization of the collision operator around $F_{i 0}^{\mathcal{E}(0)}$. In order to get (52) we have employed that for passing trajectories

$$
\int_{0}^{2 \pi} \mathrm{d} \alpha \int_{0}^{L^{(0)}(\psi)} \frac{1}{\left|v_{\|}^{(0)}\right|} C_{i i}^{\mathcal{E}, \ell(0)}\left[g_{i}^{(1)}\right] \mathrm{d} l=0 .
$$

This is obtained by noting condition (51) and by using that, due to (9), $\sum_{\sigma} \int_{0}^{L^{(0)}(\psi)}\left|v_{\|}^{(0)}\right|^{-1} C_{i i}^{\mathcal{E}, \ell(0)}[\cdot] \mathrm{d} l$ is an operator whose coefficients are independent of $\alpha$ when acting on functions independent of $l$.

The $O(\delta)$ terms of $(33)$ yield

$$
-\partial_{\psi} J^{(0)} \partial_{\alpha} F_{i 0}^{\mathcal{E}(1)}+\partial_{\alpha} J^{(1)} \Upsilon_{i}^{\mathcal{E}} F_{i 0}^{\mathcal{E}(0)}=\sum_{\sigma} \frac{Z_{i} e \Psi_{t}^{\prime}}{m_{i} c} \int_{l_{b_{10}}}^{l_{b_{20}}} \frac{\mathrm{d} l}{\left|v_{\|}^{(0)}\right|} C_{i i}^{\mathcal{E}, \ell(0)}\left[F_{i 0}^{\mathcal{E}(1)}\right] \text {, }
$$

where

$$
\Upsilon_{i}^{\mathcal{E}}=\frac{n_{i}^{\prime}}{n_{i}}+\frac{T_{i}^{\prime}}{T_{i}}\left(\frac{m_{i}\left(\mathcal{E}-Z_{i} e \varphi_{0} / m_{i}\right)}{T_{i}}-\frac{3}{2}\right)+\frac{Z_{i} e \varphi_{0}^{\prime}}{T_{i}} .
$$

Next, we show that $h_{i}^{(1)}$ can be set equal to zero. We integrate (55) over $\alpha$, which gives

$\sum_{\sigma} \int_{0}^{2 \pi} \mathrm{d} \alpha \int_{l_{b_{10}}}^{l_{b_{20}}} \frac{1}{\left|v_{\|}^{(0)}\right|} C_{i i}^{\mathcal{E}, \ell(0)}\left[h_{i}^{(1)}\right] \mathrm{d} l=0$. 
In order to obtain (57) we have used that in the trapped region

$\sum_{\sigma} \int_{0}^{2 \pi} \mathrm{d} \alpha \int_{l_{b_{10}}}^{l_{b_{20}}} \frac{1}{\left|v_{\|}^{(0)}\right|} C_{i i}^{\mathcal{E}, \ell(0)}\left[g_{i}^{(1)}\right] \mathrm{d} l=0$.

This can be deduced by recalling (51) and by noting that, due to (9), $\sum_{\sigma} \int_{l_{b_{10}}}^{l_{b_{20}}}\left|v_{\|}^{(0)}\right|^{-1} C_{i i}^{\mathcal{E}, \ell(0)}[\cdot] \mathrm{d} l$ is an operator with coefficients independent of $\alpha$ when acting on functions independent of $l$. Multiplying (52) and (57) by $-h_{i}^{(1)} / F_{i 0}^{\mathcal{E}(0)}$, integrating over velocity space and applying again an entropy-production argument, we find that $h_{i}^{(1)}$ has to be a Maxwellian distribution with zero flow, and independent of $\alpha$ and $l$. Thus, it can be absorbed in the definition of $F_{i 0}^{\mathcal{E}(0)}$ and, from here on, we can assume

$$
h_{i}^{(1)} \equiv 0
$$

without loss of generality.

Then, we only need to determine $g_{i}^{(1)}$, which is found from (55) by setting $h_{i}^{(1)}$ equal to zero. Namely,

$-\partial_{\psi} J^{(0)} \partial_{\alpha} g_{i}^{(1)}+\partial_{\alpha} J^{(1)} \Upsilon_{i}^{\mathcal{E}} F_{i 0}^{\mathcal{E}(0)}=\sum_{\sigma} \frac{Z_{i} e \Psi_{t}^{\prime}}{m_{i} c} \int_{l_{b_{10}}}^{l_{b_{20}}} \frac{\mathrm{d} l}{\left|v_{\|}^{(0)}\right|} C_{i i}^{\mathcal{E}, \ell(0)}\left[g_{i}^{(1)}\right]$.

It is obvious, but still worth pointing out, that when $\rho_{i *} \ll \nu_{i *} \ll 1$ the first term in (60) can be neglected and one recovers the equation for the dominant piece of the distribution function in the $1 / \nu$ regime of a stellarator close to omnigeneity with a non-omnigeneous perturbation that has small gradients [14].

Note that the orbit integrations in (43) and (60) only involve $B_{0}$ and $\varphi_{0}$. We use this fact to employ, in what follows, the coordinates

$$
\begin{aligned}
v & =\sqrt{2\left(\mathcal{E}-Z_{i} e \varphi_{0}(\psi) / m_{i}\right)}, \\
\lambda & =\frac{\mu}{\mathcal{E}-Z_{i} e \varphi_{0}(\psi) / m_{i}},
\end{aligned}
$$

in which the equations become simpler. We will not change the names of the functions $v_{\|}^{(0)}, \partial_{\psi} J^{(0)}, J^{(1)}$ and $g_{i}^{(1)}$ but assume that they are expressed in coordinates $v$ and $\lambda$. Let us be explicit to avoid any confusion. From here on, by $\partial_{\psi} J^{(0)}$ and $J^{(1)}$ we understand

$$
\partial_{\psi} J^{(0)}=-\int_{l_{b_{10}}}^{l_{b_{20}}} \frac{\lambda v \partial_{\psi} B_{0}+2 Z_{i} e /\left(m_{i} v\right) \varphi_{0}^{\prime}}{\sqrt{1-\lambda B_{0}}} \mathrm{~d} l
$$

and

$$
J^{(1)}=-\int_{l_{b_{10}}}^{l_{b_{20}}} \frac{\lambda v B_{1}+2 Z_{i} e /\left(m_{i} v\right) \varphi^{(1)}}{\sqrt{1-\lambda B_{0}}} \mathrm{~d} l .
$$

In these coordinates the equation for $g_{i}^{(1)}$ reads, to the relevant order in $\delta$,

$-\partial_{\psi} J^{(0)} \partial_{\alpha} g_{i}^{(1)}+\partial_{\alpha} J^{(1)} \Upsilon_{i} F_{i 0}=\sum_{\sigma} \frac{Z_{i} e \Psi_{t}^{\prime}}{m_{i} c} \int_{l_{b_{10}}}^{l_{b_{20}}} \frac{\mathrm{d} l}{\left|v_{\|}^{(0)}\right|} C_{i i}^{\ell(0)}\left[g_{i}^{(1)}\right]$,

where

$F_{i 0}(\psi, v)=n_{i}(\psi)\left(\frac{m_{i}}{2 \pi T_{i}(\psi)}\right)^{3 / 2} \exp \left(-\frac{m_{i} v^{2}}{2 T_{i}(\psi)}\right)$, 
$\Upsilon_{i}=\frac{n_{i}^{\prime}}{n_{i}}+\frac{T_{i}^{\prime}}{T_{i}}\left(\frac{m_{i} v^{2}}{2 T_{i}}-\frac{3}{2}\right)+\frac{Z_{i} e \varphi_{0}^{\prime}}{T_{i}}$

and $C_{i i}^{\ell(0)}$ is the linearized collision operator corresponding to $B_{0}$ written in coordinates $v$ and $\lambda$, given in [14].

Noting that $\int(\cdot) \mathrm{d}^{2} S \equiv \int B^{-1}|\nabla \psi|(\cdot) \mathrm{d} \alpha \mathrm{d} l$ and recalling (35), the energy flux (1) can be written as

$Q_{i}=\frac{\pi m_{i}^{2} c \delta^{2}}{Z_{i} e} \int_{0}^{\infty} \mathrm{d} v v^{3}\left(\frac{v^{2}}{2}+\frac{Z_{i} e \varphi_{0}}{m_{i}}\right) \int_{1 / B_{0, \max }}^{1 / B_{0, \min }} \mathrm{d} \lambda \int_{0}^{2 \pi} \mathrm{d} \alpha \partial_{\alpha} J^{(1)} g_{i}^{(1)}$,

where $B_{0, \min }$ and $B_{0, \max }$ are the minimum and maximum values of $B_{0}$ on the flux surface, respectively. The adiabatic response is absent from (67) because its contribution vanishes (the same can be said about its contribution to the particle flux), as shown in Appendix B.

In particular, we have shown that $Q_{i}$ is proportional to $\delta^{2}$, the square of the size of the deviation from omnigeneity. In Section 4 we expand (64) for $\nu_{i *} \ll \rho_{i *}$ and give the expressions for (67) in such collisionality regimes.

\section{Solution of the drift-kinetic equation (64) when $\nu_{i *} \ll \rho_{i *}$}

Let us define the precession frequency due to the tangential drift

$$
\omega_{\alpha}(\psi, v, \lambda):=\frac{m_{i} c}{Z_{i} e \Psi_{t}^{\prime} \tau_{b}^{(0)}} \partial_{\psi} J^{(0)}
$$

where

$$
\tau_{b}^{(0)}(\psi, v, \lambda)=\frac{2}{v} \int_{l_{b_{10}}}^{l_{b_{20}}} \frac{\mathrm{d} l}{\sqrt{1-\lambda B_{0}(\psi, \alpha, l)}}
$$

is the time that a particle trapped in $\mathbf{B}_{0}$ takes to complete its orbit. Note that $\tau_{b}^{(0)}$ does not depend on $\alpha$ due to property (9), and therefore $\omega_{\alpha}$ is also independent of $\alpha$.

Typically, $\omega_{\alpha} \sim \rho_{i *} v_{t i} / L_{0}$, and equation (64) is solved by expanding in $\nu_{i i} / \omega_{\alpha} \sim$ $\nu_{i *} / \rho_{i *} \ll 1$. We use the notation

$$
g_{i}^{(1)}=g_{0}+g_{1}+O\left(\left(\nu_{i i} / \omega_{\alpha}\right)^{2} F_{i 0}\right),
$$

where $g_{1} / g_{0} \sim O\left(\nu_{i i} / \omega_{\alpha}\right)$ and $g_{0} \sim F_{i 0}$.

To lowest order in the $\nu_{i i} / \omega_{\alpha}$ expansion, equation (64) gives

$\partial_{\alpha} g_{0}=\frac{\partial_{\alpha} J^{(1)}}{\partial_{\psi} J^{(0)}} \Upsilon_{i} F_{i 0}$

The solution of (71), choosing $\int_{0}^{2 \pi} g_{0} \mathrm{~d} \alpha=0$, is

$$
g_{0}=\frac{1}{\partial_{\psi} J^{(0)}}\left(J^{(1)}-\frac{1}{2 \pi} \int_{0}^{2 \pi} J^{(1)} \mathrm{d} \alpha\right) \Upsilon_{i} F_{i 0}
$$

Using that

$$
\left(J^{(1)}-\frac{1}{2 \pi} \int_{0}^{2 \pi} J^{(1)} \mathrm{d} \alpha\right) \partial_{\alpha} J^{(1)}=\frac{1}{2} \partial_{\alpha}\left[\left(J^{(1)}-\frac{1}{2 \pi} \int_{0}^{2 \pi} J^{(1)} \mathrm{d} \alpha\right)^{2}\right]
$$


one proves that (72) does not contribute to (67). The next order terms of (64) in the $\nu_{i i} / \omega_{\alpha}$ expansion yield

$\partial_{\alpha} g_{1}=-\frac{1}{\omega_{\alpha}} \overline{C_{i i}^{\ell(0)}\left[g_{0}\right]}$,

where we have introduced a convenient notation for the orbit average,

$$
\overline{(\cdot)}=\frac{1}{\tau_{b}^{(0)} v} \sum_{\sigma} \int_{l_{b_{10}}}^{l_{b_{20}}}(\cdot) \frac{\mathrm{d} l}{\sqrt{1-\lambda B_{0}(\psi, \alpha, l)}} .
$$

Thus,

$g_{1}=-\frac{1}{\omega_{\alpha}} \int^{\alpha} \overline{C_{i i}^{\ell(0)}\left[g_{0}\right]} \mathrm{d} \alpha^{\prime}$,

where the lower limit of the integral is selected so that $\int_{0}^{2 \pi} g_{1} \mathrm{~d} \alpha=0$.

When plugged into (67), this piece of the distribution function gives a scaling

$$
Q_{i} \sim \delta^{2} \frac{\nu_{i i}}{\omega_{\alpha}} \rho_{i *} n_{i} T_{i} v_{t i} S_{\psi}
$$

for the energy flux. However, this is not the dominant contribution to $Q_{i}$. It turns out that the energy flux is dominated by two small collisional layers that appear where (72) is not a good approximation to $g_{i}^{(1)}$. This happens near the boundary between trapped and passing particles, and also near points where $\partial_{\psi} J^{(0)}=0$. We study these layers in subsections 4.1 and 4.2 .

Finally, we advance a more subtle point. The necessity of solving the layers is not only tied to the calculation of transport fluxes. It is clear that one cannot say that the drift-kinetic equation has been completely solved unless $\varphi^{(1)}$ is known, because the latter enters the source term of the former via $\partial_{\alpha} J^{(1)}$. However, so far, $\varphi^{(1)}$ has not been found. It has to be determined from the quasineutrality equation (37). Expanding (37) in $\delta$ and choosing $\varphi^{(1)}$ such that it has vanishing flux-surface average, we obtain the equation that determines the tangential electric field

$$
\left(\frac{Z_{i}}{T_{i}}+\frac{1}{T_{e}}\right) \varphi^{(1)}=\frac{2 \pi}{e n_{i}} \int_{0}^{\infty} \mathrm{d} v \int_{B_{0, \max }^{-1}}^{B^{-1}} \mathrm{~d} \lambda \frac{v^{3} B_{0}}{\left|v_{\|}^{(0)}\right|} g_{i}^{(1)} .
$$

Here, we have used that in $\{v, \lambda, \sigma, \gamma\}$ coordinates $\int(\cdot) \mathrm{d}^{3} v \equiv \sum_{\sigma} \int(\cdot) v^{3} B /\left(2\left|v_{\|}\right|\right) \mathrm{d} v \mathrm{~d} \lambda \mathrm{d} \gamma$ and that $g_{i}^{(1)}$ vanishes in the passing region, so that the integral on the right side of $(78)$ is taken only over trapped trajectories. Note that (78) is consistent with the vanishing of the flux-surface average of $\varphi^{(1)}$ and with condition (51).

We will prove later on (see subsection 4.2.1) that the layer analyzed in subsection 4.2 contributes to the quasineutrality equation as much as $g_{0}$, in general. Hence, to treat this layer, we need to calculate $\varphi^{(1)}$ self-consistently.

\subsection{Layer around the boundary between trapped and passing particles: the $\sqrt{\nu}$ regime}

Recall that $g_{i}^{(1)} \equiv 0$ in the passing region. The value of $g_{i}^{(1)}$ at the boundary of the trapped region is given by $g_{+}:=g_{0}\left(\lambda_{c}\right) \neq 0$, with $\lambda_{c}=1 / B_{0, \max }$ and $g_{0}$ given by (72) $\boldsymbol{q}$.

I Sometimes, in order to ease the notation, we will omit some of the arguments of the functions. For example, in this section it will be common to display only the dependences on $\lambda$. 
Then, the distribution function is not continuous. This discontinuity comes from an incorrect treatment of the region around the interface between passing and trapped particles. More specifically, it is the consequence of dropping the collision term in that region. Usually, this indicates [34] that there is a small layer in a neighborhood of $\lambda_{c}$ where the distribution function develops large variations in $\lambda$, and neglecting the collision term is not correct. In the standard language of boundary-layer theory $g_{0}$ is the outer solution, and the inner solution of the boundary layer, that we will denote by $g_{\mathrm{bl}}$, remains to be found.

We have to replace (70) by

$$
g_{i}^{(1)}=g_{0}+g_{\mathrm{bl}}+\ldots,
$$

where $g_{\mathrm{bl}}$ satisfies the equation

$\omega_{\alpha} \partial_{\alpha} g_{\mathrm{bl}}+\overline{C_{i i}^{\ell(0)}\left[g_{\mathrm{bl}}\right]}=-\overline{C_{i i}^{\ell(0)}\left[g_{0}\right]}$.

The collision operator acting on $g_{0}$ has been included on the right-hand side of the previous equation because very close to $\lambda_{c}$ the function $g_{0}$ varies fast with $\lambda$, and the right side of (80) actually diverges at $\lambda_{c}$, as we will see below.

Equation (80) must be solved between $\lambda=\lambda_{c}$ and a value of $\lambda-\lambda_{c}>0$ sufficiently large that $g_{\mathrm{bl}}$ becomes small. Denote the width of the layer in the coordinate $\lambda$ by $\Delta_{\lambda}^{\sqrt{\nu}} \ll B_{0}^{-1}$ (its typical size is deduced below; see equation (112)) and let $K$ be a constant that satisfies $K \gg 1$ and $K \Delta_{\lambda}^{\sqrt{\nu}} \ll B_{0}^{-1}$. Then, equation (80) is viewed as an equation in the interval $\lambda \in\left[\lambda_{c}, \lambda_{K}\right]$, with $\lambda_{K}-\lambda_{c} \sim K \Delta_{\lambda}^{\sqrt{\nu}}$. The boundary conditions are $g_{\mathrm{bl}}\left(\lambda_{c}\right)=-g_{+}$and $g_{\mathrm{bl}}\left(\lambda_{K}\right)=0$. At the end of this subsection we will conclude that the solution is asymptotically independent of $K$, as it should be.

Due to the boundary condition $g_{\mathrm{bl}}\left(\lambda_{c}\right)=-g_{+}$, we know that $g_{\mathrm{bl}} \sim g_{0}$. In addition, if the two terms on the left side of (80) are to be comparable in size, then the support of $g_{\mathrm{bl}}$ (that is, the size of the boundary layer $\Delta_{\lambda}^{\sqrt{\nu}}$ ) has to be sufficiently small for the pitch angle scattering piece of the collision operator on the left side of (80),

$$
C_{i i}^{\ell(0)}\left[g_{\mathrm{bl}}\right]=\frac{\nu_{\lambda} v_{\|}^{(0)}}{v^{2} B_{0}} \partial_{\lambda}\left(v_{\|}^{(0)} \lambda \partial_{\lambda} g_{\mathrm{bl}}\right)+\ldots,
$$

to dominate. Here,

$\nu_{\lambda}(v)=\frac{8 \pi n_{i} Z_{i}^{4} e^{4} \ln \Lambda}{m_{i}^{2} v^{3}}\left[\operatorname{erf}\left(\sqrt{m_{i} v^{2} /\left(2 T_{i}\right)}\right)-\chi\left(\sqrt{m_{i} v^{2} /\left(2 T_{i}\right)}\right)\right]$

is the pitch angle scattering frequency, $\ln \Lambda$ is the Coulomb logarithm, $\chi(x)=[\operatorname{erf}(x)-$ $\left.(2 x / \sqrt{\pi}) \exp \left(-x^{2}\right)\right] /\left(2 x^{2}\right)$ and $\operatorname{erf}(x)=(2 / \sqrt{\pi}) \int_{0}^{x} \exp \left(-t^{2}\right) \mathrm{d} t$ is the error function.

In the boundary layer the pitch-angle scattering operator on the right side of (81) is dominated by the piece that involves $\partial_{\lambda}^{2} g_{\mathrm{bl}}$; i.e. the term that contains $\partial_{\lambda}\left(v_{\|}^{(0)} \lambda\right) \partial_{\lambda} g_{\mathrm{bl}}$ is small. The same happens for the right side of (80) close to $\lambda_{c}$, as will be justified below. Therefore, (80) can be approximated by

$\omega_{\alpha} \partial_{\alpha} g_{\mathrm{bl}}+\nu_{\lambda} \lambda \overline{B_{0}^{-1}\left(1-\lambda B_{0}\right)} \partial_{\lambda}^{2} g_{\mathrm{bl}}=-\nu_{\lambda} \lambda \overline{B_{0}^{-1}\left(1-\lambda B_{0}\right)} \partial_{\lambda}^{2} g_{0}$,

where again the coefficient multiplying $\partial_{\lambda}^{2} g_{\mathrm{bl}}$ and $\partial_{\lambda}^{2} g_{0}$ does not depend on $\alpha$ due to (9). 
Due to the smallness of the boundary layer we can approximate this equation further by taking $\lambda=\lambda_{c}$ in most terms; that is, equation (83) becomes

$$
\partial_{\psi} J^{(0)} \partial_{\alpha} g_{\mathrm{bl}}+\nu_{\lambda} \xi \partial_{\lambda}^{2} g_{\mathrm{bl}}=-\nu_{\lambda} \xi \partial_{\lambda}^{2} g_{0}
$$

where

$\xi(\psi, v):=\frac{Z_{i} e \Psi_{t}^{\prime}}{m_{i} c} \frac{2 \lambda_{c}}{v} \int_{l_{b_{10}}}^{l_{b_{20}}} B_{0}^{-1} \sqrt{1-\lambda_{c} B_{0}(\psi, \alpha, l)} \mathrm{d} l$.

The dependence of $\partial_{\psi} J^{(0)}$ on $\lambda$ cannot be neglected because $\partial_{\psi} J^{(0)}(\psi, v, \lambda)$ diverges when $\lambda \rightarrow \lambda_{c}$. The point $\lambda=\lambda_{c}$ is a singular point of the differential equation (84) and requires a careful analysis. The right-hand side of (84) also diverges at $\lambda_{c}$, as pointed out above. We proceed to explain how these divergences emerge.

In Appendix $\mathrm{C}$ we show that the asymptotic expansion of $\partial_{\psi} J^{(0)}$ for small $\lambda-\lambda_{c}$ (with $\lambda>\lambda_{c}$ ) is of the form ${ }^{+}$

$$
\partial_{\psi} J^{(0)}=a_{1} \ln \left(B_{0, \max }\left(\lambda-\lambda_{c}\right)\right)+a_{2}+O\left(v_{t i} L_{0} B_{0}\left(\lambda-\lambda_{c}\right) / \psi\right),
$$

where

$$
a_{1}=\sqrt{\frac{1}{2 \lambda_{c}}} \sum_{k=1}^{2} \frac{\lambda_{c} v \partial_{\psi} B_{0}\left(l_{M, k}\right)+2 Z_{i} e /\left(m_{i} v\right) \partial_{\psi} \varphi_{0}}{\sqrt{\left|\partial_{l}^{2} B_{0}\left(l_{M, k}\right)\right|}}
$$

and the values $l_{M, k}$, for $k=1,2$, locate two consecutive absolute maxima of $B_{0}$ when moving along the field line.

The coefficient $a_{2}$ can be computed from the relation

$$
a_{2}=\lim _{\lambda \rightarrow \lambda_{c}}\left(\partial_{\psi} J^{(0)}-a_{1} \ln \left(B_{0, \max }\left(\lambda-\lambda_{c}\right)\right)\right) .
$$

Defining $\tilde{a}_{2}$ by the relation

$$
a_{2}=a_{1} \ln \left(B_{0, \max }^{-1} \tilde{a}_{2}\right),
$$

one can recast (86) in the more convenient way

$$
\partial_{\psi} J^{(0)}=a_{1} \ln \left(\tilde{a}_{2}\left(\lambda-\lambda_{c}\right)\right)+O\left(v_{t i} L_{0} B_{0}\left(\lambda-\lambda_{c}\right) / \psi\right) .
$$

Analogously, the asymptotic expansion of $J^{(1)}$ yields

$$
J^{(1)}=c_{1} \ln \left(B_{0, \max }\left(\lambda-\lambda_{c}\right)\right)+c_{2}+O\left(v_{t i} L_{0} B_{0}\left(\lambda-\lambda_{c}\right)\right),
$$

where

$$
c_{1}=\sqrt{\frac{1}{2 \lambda_{c}}} \sum_{k=1}^{2} \frac{\lambda_{c} v B_{1}\left(l_{M, k}\right)+2 Z_{i} e /\left(m_{i} v\right) \varphi_{1}\left(l_{M, k}\right)}{\sqrt{\left|\partial_{l}^{2} B_{0}\left(l_{M, k}\right)\right|}}
$$

and

$$
c_{2}=\lim _{\lambda \rightarrow \lambda_{c}}\left(J^{(1)}-c_{1} \ln \left(B_{0, \max }\left(\lambda-\lambda_{c}\right)\right)\right) .
$$

+ An identical calculation to the one carried out in Appendix $\mathrm{C}$ for $\partial_{\psi} J^{(0)}$ shows that $\tau_{b}^{(0)}$ also diverges logarithmically when $\lambda \rightarrow \lambda_{c}$. This is not a problem in order to define the orbit-averaged drift-kinetic equation in the boundary layer because the number of particles for which $\nu_{i i} \tau_{b}^{(0)} \gg 1$ is exponentially small, $O\left(\exp \left(-1 / \nu_{i *}\right)\right)$, and therefore negligible in an asymptotic expansion in small $\nu_{i *}$. 
We rewrite $(91)$ as

$$
J^{(1)}=c_{1} \ln \left(\tilde{a}_{2}\left(\lambda-\lambda_{c}\right)\right)+\tilde{c}_{2}+O\left(v_{t i} L_{0} B_{0}\left(\lambda-\lambda_{c}\right)\right),
$$

with

$$
\tilde{c}_{2}=c_{2}-c_{1} \ln \left(B_{0, \max }^{-1} \tilde{a}_{2}\right)
$$

Using (90) and (94), equation (84) becomes

$$
\widehat{\partial_{\psi} J^{(0)}} \partial_{\alpha} g_{\mathrm{bl}}+\nu_{\lambda} \xi \partial_{\lambda}^{2} g_{\mathrm{bl}}=-\nu_{\lambda} \xi \partial_{\lambda}^{2} \widehat{g_{0}},
$$

where

$$
\begin{aligned}
& \widehat{\partial_{\psi} J^{(0)}}=a_{1} \ln \left(\tilde{a}_{2}\left(\lambda-\lambda_{c}\right)\right) \\
& \widehat{g_{0}}=\frac{1}{\widehat{\partial_{\psi} J^{(0)}}}\left(\widehat{J^{(1)}}-\frac{1}{2 \pi} \int_{0}^{2 \pi} \widehat{J^{(1)}} \mathrm{d} \alpha\right) \Upsilon_{i} F_{i 0}
\end{aligned}
$$

and

$$
\widehat{J^{(1)}}=c_{1} \ln \left(\tilde{a}_{2}\left(\lambda-\lambda_{c}\right)\right)+\tilde{c}_{2} .
$$

That is, (96) is obtained from (84) by keeping only the dominant terms in the asymptotic expansions of $\partial_{\psi} J^{(0)}$ and $J^{(1)}$ near $\lambda_{c}$. It is clear from (97), (98) and (99) that the right side of (96) diverges for $\lambda \rightarrow \lambda_{c}$. It is also obvious that, whereas both $\partial_{\psi} J^{(0)}$ and $J^{(1)}$ diverge at $\lambda_{c}, g_{+}:=g_{0}\left(\lambda_{c}\right)$ is finite, as it should be.

The solution of equation (96) is more easily found by first expanding $g_{\mathrm{bl}}$ in Fourier modes with respect to the coordinate $\alpha$. Define $g_{\mathrm{bl}, n}, g_{0, n}$ and $g_{+, n}$ by the relations

$$
\begin{aligned}
& g_{\mathrm{bl}}(\alpha, \lambda)=\sum_{n=-\infty}^{\infty} g_{\mathrm{bl}, n}(\lambda) e^{i n \alpha}, \\
& \widehat{g}_{0}(\alpha, \lambda)=\sum_{n=-\infty}^{\infty} g_{0, n}(\lambda) e^{i n \alpha} \\
& g_{+}(\alpha)=\sum_{n=-\infty}^{\infty} g_{+, n} e^{i n \alpha} .
\end{aligned}
$$

The Fourier coefficients $g_{\mathrm{bl}, 0}, g_{0,0}$ and $g_{+, 0}$ are equal to zero because of condition (51). Here, we have stressed the $\alpha$ and $\lambda$ dependence although $g_{\mathrm{bl}}, \widehat{g_{0}}$ and $g_{+}$also depend on $\psi$ and $v$. Then, (96) transforms into the set of ordinary differential equations

$$
\text { in } \widehat{\partial_{\psi} J^{(0)}} g_{\mathrm{bl}, n}+\nu_{\lambda} \xi \partial_{\lambda}^{2} g_{\mathrm{bl}, n}=-\nu_{\lambda} \xi \partial_{\lambda}^{2} g_{0, n}
$$

that must be solved with the boundary conditions

$$
g_{\mathrm{bl}, n}\left(\lambda_{c}\right)=-g_{+, n}
$$

and

$$
g_{\mathrm{bl}, n}\left(\lambda_{K}\right)=0
$$


At this point, we can explain why equation (101) has not been extended up to $\lambda=\infty$ from the beginning. The reason is that there always exists a sufficiently large value of $\lambda$ such that the truncation $\widehat{\partial_{\psi} J^{(0)}}$ vanishes even though $\partial_{\psi} J^{(0)}$ may never vanish. We will see in subsection 4.2 that points where $\partial_{\psi} J^{(0)}=0$ correspond to another layer that, typically, gives non-negligible transport. To avoid points where $\widehat{\partial_{\psi} J^{(0)}}=0$, we have imposed the boundary condition of (101) at a finite value of $\lambda_{K}$, with $\lambda_{K}-\lambda_{c} \sim K \Delta_{\lambda}^{\sqrt{\nu}}$. We must choose $K$ such that $\widehat{\partial_{\psi} J^{(0)}}$ does not vanish for $\lambda \in\left[\lambda_{c}, \lambda_{K}\right]$; i.e. such that $\left|\ln \left(\tilde{a_{2}}\left(\lambda-\lambda_{c}\right)\right)\right| \geq\left|\ln \left(\tilde{a_{2}}\left(\lambda_{K}-\lambda_{c}\right)\right)\right|$ for $\lambda \in\left[\lambda_{c}, \lambda_{K}\right]$.

The behavior of (101) in a vicinity of the singular point $\lambda=\lambda_{c}$ is analyzed in Appendix D, where it is proven that the equation possesses solutions compatible with (102).

We also expand $\widehat{J^{(1)}}$ in Fourier modes,

$$
\widehat{J^{(1)}}(\psi, \alpha, v, \lambda)=\sum_{n=-\infty}^{\infty} \widehat{J}^{(1)}(\psi, v, \lambda) e^{i n \alpha},
$$

where

$$
\widehat{J(1)}_{n}=c_{1, n} \ln \left(\tilde{a}_{2}\left(\lambda-\lambda_{c}\right)\right)+\tilde{c}_{2, n}
$$

and where $c_{1, n}$ and $\tilde{c}_{2, n}$ are the coefficients of the Fourier expansions of $c_{1}$ and $\tilde{c}_{2}$. Employing the solution for $g_{\mathrm{bl}}$, we find that the contribution of the boundary layer to the right side of $(67)$ is

$Q_{i, \sqrt{\nu}}=-\frac{2 \pi^{2} m_{i}^{2} c \delta^{2}}{Z_{i} e} \sum_{n=-\infty}^{\infty} i n \int_{0}^{\infty} \mathrm{d} v v^{3}\left(\frac{v^{2}}{2}+\frac{Z_{i} e \varphi_{0}}{m_{i}}\right) \int_{\lambda_{c}}^{\lambda_{K}} \mathrm{~d} \lambda \widehat{J^{(1)}}{ }_{-n} g_{\mathrm{bl}, n}$.

We proceed to show the scaling of (106) with the square root of the collisionality, to which the $\sqrt{\nu}$ regime owes its name. We will also prove that the scaling with the square root of the collisionality must actually be corrected by a logarithm due to the logarithmic singularities in (101). Finally, we will show that the solution is independent of the constant $K$ as long as $\left|\ln \left(\tilde{a_{2}}\left(\lambda_{K}-\lambda_{c}\right)\right)\right|$ is sufficiently large.

In (101), we perform the change of coordinate

$$
z=\sqrt{\frac{1}{\Delta^{2}} \ln \left(\frac{1}{\tilde{a}_{2}^{2} \Delta^{2}}\right)}\left(\lambda-\lambda_{c}\right)
$$

where

$$
\Delta=\left(\frac{\nu_{\lambda} \xi}{\left|n a_{1}\right|}\right)^{1 / 2}
$$

Then, equation (101) becomes

$i \frac{n a_{1}}{\left|n a_{1}\right|}\left[\frac{\ln z}{\ln \left(1 /\left(\tilde{a}_{2} \Delta\right)^{2}\right)}-\frac{1}{2}\left(1+\frac{\ln \left[\ln \left(1 /\left(\tilde{a}_{2} \Delta\right)^{2}\right)\right]}{\ln \left(1 /\left(\tilde{a}_{2} \Delta\right)^{2}\right)}\right)\right] g_{\mathrm{bl}, n}+\partial_{z}^{2} g_{\mathrm{bl}, n}=\partial_{z}^{2} g_{0, n}$,

with

$g_{0, n}=\frac{1}{a_{1}}\left[c_{1, n}-\frac{2 \tilde{c}_{2, n}}{\left.\ln \left(1 /\left(\tilde{a}_{2}^{2} \Delta^{2}\right)\right)+\ln \left[\ln \left(1 /\left(\tilde{a}_{2} \Delta\right)^{2}\right)\right)\right]-2 \ln z}\right] \Upsilon_{i} F_{i 0}$. 
Employing that $z \sim 1$ in the layer and performing an expansion in the small quantity $1 / \ln \left(1 /\left(\tilde{a}_{2} \Delta\right)^{2}\right) \ll 1$, one obtains to lowest order

$$
-\frac{i}{2} \frac{n a_{1}}{\left|n a_{1}\right|} g_{\mathrm{bl}, n}+\partial_{z}^{2} g_{\mathrm{bl}, n}=0
$$

where we have used, in particular, that the right side of (109) is small in $1 / \ln \left(1 /\left(\tilde{a}_{2} \Delta\right)^{2}\right)$, as can be deduced by inspecting (110). Equation (111) has an exponentially decaying solution with a characteristic width $\Delta_{z} \sim 1$. Using (107) to go back to the original coordinate $\lambda$, we find that the width of the layer in $\lambda$ is

$$
\Delta_{\lambda}^{\sqrt{\nu}} \sim\left(\frac{\nu_{\lambda} \xi}{\left|n a_{1}\right|}\right)^{1 / 2} \frac{1}{\sqrt{\ln \left(1 /\left(\tilde{a}_{2} \Delta\right)^{2}\right)}} .
$$

Therefore, not even to lowest order in $1 / \ln \left(1 /\left(\tilde{a}_{2} \Delta\right)^{2}\right) \ll 1$ does the width of the layer scale exactly with the square root of the collision frequency. Although the logarithmic corrections do not change the qualitative features of this collisionality regime, they must be accounted for in order to have accurate results for the neoclassical fluxes. Noting the asymptotic expression (105), and using the change of coordinate (107) to rewrite the right side of (106), we find that the size of $Q_{i, \sqrt{\nu}}$ is

$$
Q_{i, \sqrt{\nu}} \sim \delta^{2} \frac{\nu_{i i}^{1 / 2}}{\omega_{\alpha}^{3 / 2}} \sqrt{\ln \left(\omega_{\alpha} / \nu_{i i}\right)} \rho_{i *}^{2} n_{i} T_{i} v_{t i}^{2} L_{0}^{-1} S_{\psi} .
$$

Finally, we point out that the expansion of (109) in the small quantity $1 / \ln \left(1 /\left(\tilde{a}_{2} \Delta\right)^{2}\right)$ can be continued to higher orders. It is straightforward to check that, to any order, the boundary condition for large $z$ (equivalently, for large $\lambda$ ) can be imposed at $z=\infty$. In other words, the solution to equation (109) is independent of $K$ when $1 / \ln \left(1 /\left(\tilde{a}_{2} \Delta\right)^{2}\right) \ll 1$.

Recall that $\varphi_{1}^{(1)}$ remains to be found. In order to write the precise form of the quasineutrality equation that determines $\varphi_{1}^{(1)}$ (given in subsection 4.2.1), we have to solve for the layer around points where $\partial_{\psi} J^{(0)}=0$. This is the subject of subsection 4.2 .

\subsection{Layer around points where $\omega_{\alpha}=0$ : the superbanana-plateau regime}

The outer solution (72) for the distribution function is correct everywhere except near the boundary between the passing and trapped regions (already treated in subsection 4.1 ) and in the neighborhood of points where $\omega_{\alpha}=0$ (equivalently, points where $\left.\partial_{\psi} J^{(0)}=0\right)$. Around these 'resonant points' the $\nu_{i i} / \omega_{\alpha} \ll 1$ expansion is not valid. This region of phase space is the subject of the present section.

In order to understand what happens in the vicinity of a point where $\omega_{\alpha}=0$, we go back to equation (64) and do not carry out the $\nu_{i i} / \omega_{\alpha} \ll 1$ expansion. That is, we consider the equation

$$
\omega_{\alpha} \partial_{\alpha} g_{i}^{(1)}+\overline{C_{i i}^{\ell(0)}\left[g_{i}^{(1)}\right]}=S
$$


with

$$
S(\psi, \alpha, v, \lambda)=\frac{m_{i} c}{Z_{i} e \Psi_{t}^{\prime} \tau_{b}^{(0)}} \partial_{\alpha} J^{(1)} \Upsilon_{i} F_{i 0}
$$

Below we will find it useful to distinguish between the contributions to $\partial_{\alpha} J^{(1)}$ coming from $B_{1}$ and from $\varphi^{(1)}$. Defining

$$
J_{B}^{(1)}=-\lambda v \int_{l_{b_{10}}}^{l_{b_{20}}} \frac{B_{1}}{\sqrt{1-\lambda B_{0}}} \mathrm{~d} l
$$

and

$$
J_{\varphi}^{(1)}=-\frac{2 Z_{i} e}{m_{i} v} \int_{l_{b_{10}}}^{l_{b_{20}}} \frac{\varphi^{(1)}}{\sqrt{1-\lambda B_{0}}} \mathrm{~d} l,
$$

we write $S=S_{B}+S_{\varphi}$, where

$$
S_{B}(\psi, \alpha, v, \lambda)=\frac{m_{i} c}{Z_{i} e \Psi_{t}^{\prime} \tau_{b}^{(0)}} \partial_{\alpha} J_{B}^{(1)} \Upsilon_{i} F_{i 0}
$$

and

$$
S_{\varphi}(\psi, \alpha, v, \lambda)=\frac{m_{i} c}{Z_{i} e \Psi_{t}^{\prime} \tau_{b}^{(0)}} \partial_{\alpha} J_{\varphi}^{(1)} \Upsilon_{i} F_{i 0}
$$

We call $\lambda_{r}$ the values of $\lambda$ that satisfy $\omega_{\alpha}=0$. Given an omnigeneous magnetic field $\mathbf{B}_{0}$, they are found from the equation (recall (62) and (75))

$$
\lambda_{r} \overline{\partial_{\psi} B_{0}}\left(\psi, \lambda_{r}\right)=-\frac{2 Z_{i} e \varphi_{0}^{\prime}(\psi)}{m_{i} v^{2}} .
$$

Of course, in general $\lambda_{r}$ is a function of $\psi$ and $v, \lambda_{r} \equiv \lambda_{r}(\psi, v)$. The qualitative discussion on the number of zeroes of (120) depends on the number of zeroes of (120) for the particular case of $\varphi_{0}^{\prime}=0$,

$$
\overline{\partial_{\psi} B_{0}}\left(\psi, \lambda_{r 0}\right)=0 \text {. }
$$

To fix ideas, we assume the common situation in which one, and only one value of $\lambda$ solves equation (121). In this setting, for any value of $\varphi_{0}^{\prime}$ and $v, \omega_{\alpha}$ vanishes at most for one value of $\lambda$. And for any given value of $\varphi_{0}^{\prime}$, there exists a minimum value of $v$ such that $\omega_{\alpha}=0$ for some value of $\lambda$. We denote this value of $v$ by $v_{\min }$.

Around $\lambda_{r}$,

$$
\omega_{\alpha}(\lambda)=\partial_{\lambda} \omega_{\alpha}\left(\lambda_{r}\right)\left(\lambda-\lambda_{r}\right)+O\left(\left(\lambda-\lambda_{r}\right)^{2}\right),
$$

where the dependence on $\psi$ and $v$ has been omitted for simplicity. The balance of the two terms on the left side of (114) implies that in a neighborhood of $\lambda_{r}$ of size $\Delta_{\lambda}^{\mathrm{sb}-\mathrm{p}}$,

$$
\partial_{\lambda} \omega_{\alpha}\left(\lambda_{r}\right) \Delta_{\lambda}^{\mathrm{sb}-\mathrm{p}} \sim \frac{\nu_{i i}}{B_{0}^{2}\left(\Delta_{\lambda}^{\mathrm{sb}-\mathrm{p}}\right)^{2}}
$$

Since, typically, $\partial_{\lambda} \omega_{\alpha}\left(\lambda_{r}\right) \sim \rho_{i *} B_{0} L_{0}^{-1} v_{t i}$, one finds

$$
B_{0} \Delta_{\lambda}^{\mathrm{sb}-\mathrm{p}} \sim\left(\frac{\nu_{i *}}{\rho_{i *}}\right)^{1 / 3} \ll 1 .
$$


In the particular case of a large-aspect-ratio tokamak with broken symmetry, this estimation coincides with the one obtained in [35].

Denote by $g_{\mathrm{rl}}$ the distribution function in this 'resonant layer'. The pitch-angle scattering piece of the collision operator dominates the collision term in this layer,

$$
\overline{C_{i i}^{\ell(0)}\left[g_{\mathrm{rl}}\right]}=\overline{\frac{\nu_{\lambda} v_{\|}^{(0)}}{v^{2} B_{0}} \partial_{\lambda}\left(v_{\|}^{(0)} \lambda \partial_{\lambda} g_{\mathrm{rl}}\right)}+\ldots,
$$

and, in fact, we can keep only the term involving $\partial_{\lambda}^{2} g_{\mathrm{rl}}$. Hence, in the resonant layer we write the drift kinetic equation as

$$
\partial_{\lambda} \omega_{\alpha, r}\left(\lambda-\lambda_{r}\right) \partial_{\alpha} g_{\mathrm{rl}}+\nu_{\lambda} \chi_{r} \partial_{\lambda}^{2} g_{\mathrm{rl}}=S_{B, r}+\widehat{S_{\varphi}}
$$

with

$$
\begin{aligned}
& \chi_{r}(\psi, v):=\lambda_{r} \overline{B_{0}^{-1}\left(1-\lambda_{r} B_{0}\right)} \\
& \partial_{\lambda} \omega_{\alpha, r}(\psi, v):=\partial_{\lambda} \omega_{\alpha}\left(\psi, v, \lambda_{r}(\psi, v)\right) \\
& S_{B, r}(\psi, \alpha, v):=S_{B}\left(\psi, \alpha, v, \lambda_{r}(\psi, v)\right)
\end{aligned}
$$

and

$$
\widehat{S_{\varphi}}(\psi, \alpha, v, \lambda)=\frac{m_{i} c}{Z_{i} e \Psi_{t}^{\prime} \tau_{b, r}^{(0)}} \partial_{\alpha} \widehat{J_{\varphi}^{(1)}}(\psi, \alpha, v, \lambda) \Upsilon_{i} F_{i 0}
$$

with $\tau_{b, r}^{(0)}=\tau_{b}^{(0)}\left(\psi, v, \lambda_{r}(\psi, v)\right)$ and

$$
\begin{aligned}
\widehat{J_{\varphi}^{(1)}}= & -\frac{2 Z_{i} e}{m_{i} v} \int_{\tilde{l}_{b_{10}}}^{l_{R}} \frac{\varphi^{(1)}}{\sqrt{\lambda_{r}\left|\partial_{l} B_{0}\left(l_{L}\right)\right|\left(l-l_{L}\right)-\left(\lambda-\lambda_{r}\right) B_{0}\left(l_{L}\right)}} \mathrm{d} l \\
& -\frac{2 Z_{i} e}{m_{i} v} \int_{l_{L}}^{\tilde{l}_{b_{20}}} \frac{\varphi^{(1)}}{\sqrt{\lambda_{r}\left|\partial_{l} B_{0}\left(l_{R}\right)\right|\left(l_{R}-l\right)-\left(\lambda-\lambda_{r}\right) B_{0}\left(l_{R}\right)}} \mathrm{d} l \\
& -\frac{2 Z_{i} e}{m_{i} v} \int_{l_{L}}^{l_{R}} \varphi^{(1)}\left[\frac{1}{\sqrt{1-\lambda_{r} B_{0}(l)}}-\frac{1}{\sqrt{\lambda_{r}\left|\partial_{l} B_{0}\left(l_{L}\right)\right|\left(l-l_{L}\right)}}\right. \\
& \left.-\frac{1}{\sqrt{\lambda_{r}\left|\partial_{l} B_{0}\left(l_{R}\right)\right|\left(l_{R}-l\right)}}\right] \mathrm{d} l .
\end{aligned}
$$

We have denoted by $l_{L}$ and $l_{R}$, respectively, the left and right bounce points of the orbit corresponding to $\lambda=\lambda_{r}$; i.e. the solutions for $l$ of $1-\lambda_{r} B_{0}(l)=0$. In $(131), \tilde{l}_{b_{10}}$ and $\tilde{l}_{b_{20}}$ are approximations to the exact bounce points, $l_{b_{10}}$ and $l_{b_{20}}$, given by

$$
\tilde{l}_{b_{10}}-l_{L}=\frac{B_{0}\left(l_{L}\right)}{\lambda_{r}\left|\partial_{l} B_{0}\left(l_{L}\right)\right|}\left(\lambda-\lambda_{r}\right)
$$

and

$$
l_{R}-\tilde{l}_{b_{20}}=\frac{B_{0}\left(l_{R}\right)}{\lambda_{r}\left|\partial_{l} B_{0}\left(l_{R}\right)\right|}\left(\lambda-\lambda_{r}\right)
$$

Expression (131) is an asymptotic approximation to $J_{\varphi}^{(1)}$ near the resonant value of the pitch-angle coordinate, as can be proven by using the techniques developed in [30]. 
Obviously, if $\varphi^{(1)}$ were regular everywhere, one could simply evaluate $J_{\varphi}^{(1)}$ at the resonant value $\lambda=\lambda_{r}$ (which would amount to retaining only the first term in square brackets in (131)). However, in subsections 4.2.1 and 4.2.2 we will show that if $\varphi_{0}^{\prime}$ is small then $\varphi^{(1)} \sim\left(\rho_{i *} / \nu_{i *}\right)^{1 / 6} T_{i} / e \gg T_{i} / e$ in a neighborhood of $l_{L}$ and $l_{R}$, and therefore the more elaborate asymptotic expression (131) is nedeed.

Equation (126) is viewed as a differential equation in $\lambda \in(-\infty, \infty)$ with vanishing boundary conditions at infinity. Note that a rescaling of the coordinate $\lambda$

$$
x=\left(\frac{\nu_{\lambda} \chi_{r}}{\partial_{\lambda} \omega_{\alpha, r}}\right)^{-1 / 3}\left(\lambda-\lambda_{r}\right)
$$

gives the expression

$$
\Delta_{\lambda}^{\mathrm{sb}-\mathrm{p}}=\left(\frac{\nu_{\lambda} \chi_{r}}{\partial_{\lambda} \omega_{\alpha, r}}\right)^{1 / 3}
$$

for the size of the layer needed to make the two terms on the left side of (126) comparable. Then, the size of the distribution function in the layer can be estimated as*

$$
g_{\mathrm{rl}} \sim \frac{S_{B, r}}{\partial_{\lambda} \omega_{\alpha, r} \Delta_{\lambda}^{\mathrm{sb}-\mathrm{p}}} \sim \frac{1}{B_{0} \Delta_{\lambda}^{\mathrm{sb}-\mathrm{p}}} F_{i 0} .
$$

Define the Fourier expansions

$$
\begin{aligned}
& g_{\mathrm{rl}}=\sum_{n=-\infty}^{\infty} g_{\mathrm{rl}, n} e^{i n \alpha}, \\
& S_{B, r}=\sum_{n=-\infty}^{\infty}\left(S_{B, r}\right)_{n} e^{i n \alpha}, \\
& \widehat{S_{\varphi}}=\sum_{n=-\infty}^{\infty}\left(\widehat{S_{\varphi}}\right)_{n} e^{i n \alpha} .
\end{aligned}
$$

The coefficient $g_{\mathrm{rl}, 0}$ vanishes due to $(51)$, and $\left(S_{B, r}\right)_{0}$ and $\left(\widehat{S_{\varphi}}\right)_{0}$ vanish due to definition (115). Inserting the expansions in (126) and noting that $\partial_{\lambda} \omega_{\alpha, r}$ and $\chi_{r}$ do not depend on $\alpha$, we find an ordinary differential equation for each mode $g_{\mathrm{rl}, n}$,

$$
i n \partial_{\lambda} \omega_{\alpha, r}\left(\lambda-\lambda_{r}\right) g_{\mathrm{rl}, n}+\nu_{\lambda} \chi_{r} \partial_{\lambda}^{2} g_{\mathrm{rl}, n}=\left(S_{B, r}\right)_{n}+\left(\widehat{S_{\varphi}}\right)_{n}
$$

In terms of the solution to this set of equations, the energy flux (67) can be written as

$$
\begin{aligned}
& Q_{i, \mathrm{sb}-\mathrm{p}}=- \frac{2 \pi^{2} m_{i}^{2} c \delta^{2}}{Z_{i} e} \sum_{n=-\infty}^{\infty} i n \int_{v_{\min }}^{\infty} \mathrm{d} v v^{3}\left(\frac{v^{2}}{2}+\frac{Z_{i} e \varphi_{0}}{m_{i}}\right) \int_{-\infty}^{\infty} \mathrm{d} \lambda\left[\left(J_{B, r}^{(1)}\right)_{-n}\right. \\
&\left.+\left(\widehat{J_{\varphi}^{(1)}}\right)_{-n}\right] g_{\mathrm{rl}, n} .
\end{aligned}
$$

* The perturbation to the Maxwellian has a size $\delta g_{i}^{(1)} \sim \delta g_{\mathrm{rl}}$ in the layer. From (136), one might be worried that the perturbation to the Maxwellian becomes larger than the Maxwellian when $\nu_{i *}<\rho_{i *} \delta^{3}$. This is not a problem, however, because the analysis in this subsection does not apply to such small values of the collisionality. This is explained in Section 6 (see equation (185)). 
Here, $\left(\widehat{J_{\varphi}^{(1)}}\right)_{n}$ are the coefficients of the Fourier expansion of $\widehat{J_{\varphi}^{(1)}}$ and $\left(J_{B, r}^{(1)}\right)_{n}$ are the coefficients of the Fourier expansion of

$$
J_{B, r}^{(1)}(\psi, \alpha, v):=J_{B}^{(1)}\left(\psi, \alpha, v, \lambda_{r}(\psi, v)\right) .
$$

As long as $v_{\min } \lesssim v_{t i}$, the typical size of the energy flux is

$$
Q_{i, \mathrm{sb}-\mathrm{p}} \sim \delta^{2} \rho_{i *} n_{i} T_{i} v_{t i} S_{\psi},
$$

which is a consequence of using (135) and (136) in (139). In particular, $Q_{i, \mathrm{sb}-\mathrm{p}}$ does not scale with any power of collisionality. This is the most characteristic feature of the superbanana-plateau regime. Below, we explain that the estimates (135), (136) and (141), that are correct for sufficiently large radial electric field (see subsection 4.2.3), must be refined by including logarithmic corrections if the radial electric field is small enough (see subsection 4.2.2). The reason is that to obtain (135), (136) and (141), we have skipped features of $\widehat{J_{\varphi}^{(1)}}$ that are important when $\varphi_{0}^{\prime}$ is small.

Before turning to deal with the quasineutrality equation in the next subsection, it is useful to identify the piece of the distribution function $g_{i}^{(1)}$ out of the resonant layer. As we pointed out, $g_{0}$ diverges when $\partial_{\psi} J^{(0)}=0$ and therefore it has to be replaced by $g_{\mathrm{rl}}$ in the layer. Sometimes, it is convenient to explicitly write $g_{i}^{(1)}$ as a sum of terms that are specifically associated to the layer and to the region external to the layer. This splitting is given by

$$
g_{i}^{(1)}=g_{0}^{\text {out }}+g_{\mathrm{rl}},
$$

where

$$
\begin{aligned}
g_{0}^{\text {out }}= & g_{0}-\frac{1}{\left(\lambda-\lambda_{r}\right) \partial_{\lambda} \partial_{\psi} J^{(0)}\left(\lambda_{r}\right)}\left(J_{B, r}^{(1)}-\frac{1}{2 \pi} \int_{0}^{2 \pi} J_{B, r}^{(1)} \mathrm{d} \alpha\right. \\
& \left.+\widehat{J_{\varphi}^{(1)}}-\frac{1}{2 \pi} \int_{0}^{2 \pi} \widehat{J_{\varphi}^{(1)}} \mathrm{d} \alpha\right) \Upsilon_{i} F_{i 0} .
\end{aligned}
$$

4.2.1. Quasineutrality equation. We are ready to write more explicitly the quasineutrality equation (78), needed to find $\varphi^{(1)}$. The solution (72) does not contribute to transport but it does contribute to (78). The component $g_{\mathrm{bl}}$, associated to the $\sqrt{\nu}$ regime, gives a negligible contribution because $g_{\mathrm{bl}} \sim F_{i 0}$ and the size of the layer is small (see (112)). However, in general, $g_{\mathrm{rl}}$ does contribute to (78) as much as $g_{0}$ (more precisely, as much as $g_{0}^{\text {out }}$, defined in (143)) due to the combination of (135) and (136).

Asymptotically, (78) reads

$$
\begin{aligned}
\left(\frac{Z_{i}}{T_{i}}\right. & \left.+\frac{1}{T_{e}}\right) \varphi^{(1)}=\frac{2 \pi}{e n_{i}} \int_{0}^{\infty} \mathrm{d} v \int_{B_{0, \text { max }}^{-1}}^{B^{-1}} \mathrm{~d} \lambda \frac{v^{3} B_{0}}{\left|v_{\|}^{(0)}\right|} g_{0}^{\text {out }} \\
& +\frac{2 \pi B_{0}}{e n_{i}} \int_{v_{\min }}^{\infty} \mathrm{d} v v^{2} \int_{-\infty}^{\lambda_{L}(l)} \mathrm{d} \lambda \frac{g_{\mathrm{rl}}}{\sqrt{\lambda_{r}\left|\partial_{l} B_{0}\left(l_{L}\right)\right|\left(l-l_{L}\right)-\left(\lambda-\lambda_{r}\right) B_{0}\left(l_{L}\right)}} \\
& +\frac{2 \pi B_{0}}{e n_{i}} \int_{v_{\min }}^{\infty} \mathrm{d} v v^{2} \int_{-\infty}^{\lambda_{R}(l)} \mathrm{d} \lambda \frac{g_{\mathrm{rl}}}{\sqrt{\lambda_{r}\left|\partial_{l} B_{0}\left(l_{R}\right)\right|\left(l_{R}-l\right)-\left(\lambda-\lambda_{r}\right) B_{0}\left(l_{R}\right)}}
\end{aligned}
$$




$$
\begin{aligned}
& +\frac{2 \pi B_{0}}{e n_{i}} \int_{v_{\min }}^{\infty} \mathrm{d} v v^{2}\left[\frac{1}{\sqrt{1-\lambda_{r} B_{0}(l)}}-\frac{1}{\sqrt{\lambda_{r}\left|\partial_{l} B_{0}\left(l_{L}\right)\right|\left(l-l_{L}\right)}}\right. \\
& \left.-\frac{1}{\sqrt{\lambda_{r}\left|\partial_{l} B_{0}\left(l_{R}\right)\right|\left(l_{R}-l\right)}}\right] \int_{-\infty}^{\infty} \mathrm{d} \lambda g_{\mathrm{rl}} .
\end{aligned}
$$

Here,

$$
\lambda_{L}(l)-\lambda_{r}=\frac{\lambda_{r}\left|\partial_{l} B_{0}\left(l_{L}\right)\right|\left(l-l_{L}\right)}{B_{0}\left(l_{L}\right)}
$$

and

$$
\lambda_{R}(l)-\lambda_{r}=\frac{\lambda_{r}\left|\partial_{l} B_{0}\left(l_{R}\right)\right|\left(l_{R}-l\right)}{B_{0}\left(l_{R}\right)} .
$$

Of course, $\lambda_{L}$ and $\lambda_{R}$ depend on $\psi$ and $v$ as well, but for brevity we have only displayed the dependence on $l$, as we often do with other functions along the paper. The necessity for the complicated asymptotic expansion employed for the factor $\left|v_{\|}^{(0)}\right|^{-1}$, instead of simply keeping the first term in square brackets in (144), can be understood by observing that such a term diverges when $l=l_{L}$. Let us discuss this in more detail.

The function $\lambda_{r}(\psi, v)$ is obtained from condition (120). For the particular case of $\varphi_{0}^{\prime}=0$, the resonant value of $\lambda$ is obtained from (121) and is denoted by $\lambda_{r 0}(\psi)$, where we have stressed that $\lambda_{r 0}$ does not depend on $v$. This will be important in what follows. The correction $\lambda_{r}-\lambda_{r 0}$ that is linear in $\varphi_{0}^{\prime}$ is found from

$$
\lambda_{r}-\lambda_{r 0}=-\frac{2 Z_{i} e \varphi_{0}^{\prime}(\psi)}{m_{i} v^{2}}\left[\overline{\partial_{\psi} B_{0}}\left(\psi, \lambda_{r 0}\right)+\lambda_{r 0} \partial_{\lambda} \overline{\partial_{\psi} B_{0}}\left(\psi, \lambda_{r 0}\right)\right]^{-1} .
$$

Defining $l_{L 0}$ and $l_{R 0}$ as the solutions for $l$ of $1-\lambda_{r 0} B_{0}(l)=0$, the corrections $l_{L}-l_{L 0}$ and $l_{R}-l_{R 0}$ are given by

$$
\begin{aligned}
& l_{L}-l_{L 0}=-\frac{2 Z_{i} e \varphi_{0}^{\prime}(\psi)}{m_{i} v^{2}} \frac{B_{0}\left(l_{L 0}\right)}{\lambda_{r 0}\left|\partial_{l} B_{0}\left(l_{L 0}\right)\right|}\left[\overline{\partial_{\psi} B_{0}}\left(\psi, \lambda_{r 0}\right)+\lambda_{r 0} \partial_{\lambda} \overline{\partial_{\psi} B_{0}}\left(\psi, \lambda_{r 0}\right)\right]^{-1}, \\
& l_{R}-l_{R 0}=\frac{2 Z_{i} e \varphi_{0}^{\prime}(\psi)}{m_{i} v^{2}} \frac{B_{0}\left(l_{R 0}\right)}{\lambda_{r 0}\left|\partial_{l} B_{0}\left(l_{R 0}\right)\right|}\left[\overline{\partial_{\psi} B_{0}}\left(\psi, \lambda_{r 0}\right)+\lambda_{r 0} \partial_{\lambda} \overline{\partial_{\psi} B_{0}}\left(\psi, \lambda_{r 0}\right)\right]^{-1} .
\end{aligned}
$$

In order to make further progress we have to give an ordering for $\varphi_{0}^{\prime}$, distinguishing the cases of small and large radial electric field as defined by the conditions

$$
\lambda_{r}-\lambda_{r 0} \sim \frac{2 Z_{i} e \varphi_{0}^{\prime}}{m_{i} v^{2} \overline{\partial_{\psi} B_{0}}\left(\psi, \lambda_{r 0}\right)} \ll \Delta_{\lambda}^{\mathrm{sb}-\mathrm{p}}
$$

and

$$
\lambda_{r}-\lambda_{r 0} \sim \frac{2 Z_{i} e \varphi_{0}^{\prime}}{m_{i} v^{2} \overline{\partial_{\psi} B_{0}}\left(\psi, \lambda_{r 0}\right)} \gg \Delta_{\lambda}^{\mathrm{sb}-\mathrm{p}}
$$

respectively. 
4.2.2. Small $\varphi_{0}^{\prime}$. Let us take the first term that contains $g_{\mathrm{rl}}$ in (144). Using (148), we can write

$$
\begin{aligned}
& \frac{2 \pi B_{0}}{e n_{i}} \int_{v_{\min }}^{\infty} \mathrm{d} v v^{2} \int_{-\infty}^{\lambda_{L}(l)} \mathrm{d} \lambda \frac{g_{\mathrm{rl}}}{\sqrt{\lambda_{r}\left|\partial_{l} B_{0}\left(l_{L}\right)\right|\left(l-l_{L}\right)-\left(\lambda-\lambda_{r}\right) B_{0}\left(l_{L}\right)}} \approx \\
& \frac{2 \pi B_{0}}{e n_{i}} \int_{v_{\min }}^{\infty} \mathrm{d} v v^{2} \int_{-\infty}^{\lambda_{L}(l)} \mathrm{d} \lambda \frac{g_{\mathrm{rl}}}{\sqrt{\lambda_{r}\left|\partial_{l} B_{0}\left(l_{L}\right)\right|\left(l-l_{L 0}-\kappa_{L}\right)-\left(\lambda-\lambda_{r}\right) B_{0}\left(l_{L}\right)}},
\end{aligned}
$$

where

$\kappa_{L}=-\frac{2 Z_{i} e \varphi_{0}^{\prime}(\psi)}{m_{i} v^{2}} \frac{B_{0}\left(l_{L 0}\right)}{\lambda_{r 0}\left|\partial_{l} B_{0}\left(l_{L 0}\right)\right|}\left[\overline{\partial_{\psi} B_{0}}\left(\psi, \lambda_{r 0}\right)+\lambda_{r 0} \partial_{\lambda} \overline{\partial_{\psi} B_{0}}\left(\psi, \lambda_{r 0}\right)\right]^{-1}$.

If (150) holds, we can set $\varphi_{0}^{\prime}=0$ in the previous expressions and find

$$
\begin{aligned}
& \frac{2 \pi B_{0}}{e n_{i}} \int_{v_{\min }}^{\infty} \mathrm{d} v v^{2} \int_{-\infty}^{\lambda_{L}(l)} \mathrm{d} \lambda \frac{g_{\mathrm{rl}}}{\sqrt{\lambda_{r}\left|\partial_{l} B_{0}\left(l_{L}\right)\right|\left(l-l_{L}\right)-\left(\lambda-\lambda_{r}\right) B_{0}\left(l_{L}\right)}}= \\
& \frac{2 \pi B_{0}}{e n_{i}} \int_{0}^{\infty} \mathrm{d} v v^{2} \int_{-\infty}^{\lambda_{L 0}(l)} \mathrm{d} \lambda \frac{g_{\mathrm{rl}}}{\sqrt{\lambda_{r 0}\left|\partial_{l} B_{0}\left(l_{L 0}\right)\right|\left(l-l_{L 0}\right)-\left(\lambda-\lambda_{r 0}\right) B_{0}\left(l_{L 0}\right)}},
\end{aligned}
$$

where $\lambda_{L 0}$ stands for the function $\lambda_{L}$ in the particular case of $\varphi_{0}^{\prime}=0$. The key observation is that the quantity under the square root on the right side of the last equation is independent of $v$. Then, at $l=l_{L 0}$, the right side of (154) becomes

$\frac{2 \pi B_{0}}{e n_{i}} \int_{0}^{\infty} \mathrm{d} v v^{2} \int_{-\infty}^{\lambda_{r} 0} \mathrm{~d} \lambda \frac{g_{\mathrm{rl}}}{\sqrt{\left(\lambda_{r 0}-\lambda\right) B_{0}\left(l_{L 0}\right)}}$.

Therefore, when $\varphi_{0}^{\prime}$ is small, the first term that contains $g_{\mathrm{rl}}$ in (144) gives a contribution to $\varphi^{(1)}$ whose typical size is

$\varphi^{(1)} \sim \frac{m_{i} v_{t i}^{5}}{e n_{i}} B_{0} \Delta_{\lambda}^{\mathrm{sb}-\mathrm{p}} g_{\mathrm{rl}} \frac{1}{\left(B_{0} \Delta_{\lambda}^{\mathrm{sb}-\mathrm{p}}\right)^{1 / 2}} \quad$ when $l-l_{L 0} \sim B_{0} \Delta_{\lambda}^{\mathrm{sb}-\mathrm{p}} L_{0}$

and

$\varphi^{(1)} \sim \frac{m_{i} v_{t i}^{5}}{e n_{i}} B_{0} \Delta_{\lambda}^{\mathrm{sb}-\mathrm{p}} g_{\mathrm{rl}} \frac{1}{\sqrt{\left(l-l_{L 0}\right) / L_{0}}} \quad$ when $l-l_{L 0} \gg B_{0} \Delta_{\lambda}^{\mathrm{sb}-\mathrm{p}} L_{0}$.

When inserted in $(131)$, this piece of $\varphi^{(1)}$ gives a contribution to $\widehat{J_{\varphi}^{(1)}}$ that scales as

$$
\widehat{J_{\varphi}^{(1)}} \sim \frac{v_{t i}^{4} L_{0}}{n_{i}} B_{0} \Delta_{\lambda}^{\mathrm{sb}-\mathrm{p}} g_{\mathrm{rl}} \ln \left(B_{0} \Delta_{\lambda}^{\mathrm{sb}-\mathrm{p}}\right) .
$$

We are ready to show why the estimates (135), (136) and (141) are not completely correct if $\varphi_{0}^{\prime}$ is small. Inspecting the size of each term in (126) and recalling (158), one concludes that the width of the layer, $\Delta_{\lambda}^{\mathrm{sb}-\mathrm{p}}$, is determined by balancing the collision term and the last term on the right side of equation (126). The result is

$$
B_{0} \Delta_{\lambda}^{\mathrm{sb}-\mathrm{p}} \sim\left(\frac{\nu_{i *}}{\rho_{i *}}\right)^{1 / 3}\left[\ln \left(\rho_{i *} / \nu_{i *}\right)\right]^{-1 / 3} .
$$


The size of the distribution function is found by balancing the two terms on the right side of (126), obtaining

$$
g_{\mathrm{rl}} \sim\left(\frac{\rho_{i *}}{\nu_{i *}}\right)^{1 / 3}\left[\ln \left(\rho_{i *} / \nu_{i *}\right)\right]^{-2 / 3} F_{i 0} .
$$

Then, the ion energy flux (139) scales as

$$
Q_{i, \mathrm{sb}-\mathrm{p}} \sim \delta^{2} \frac{\rho_{i *}}{\ln \left(\rho_{i *} / \nu_{i *}\right)} n_{i} T_{i} v_{t i} S_{\psi} .
$$

4.2.3. Large $\varphi_{0}^{\prime}$. We consider again the first term that contains $g_{\mathrm{rl}}$ in (144) and recall expressions (152) and (153). Now, assume that (151) holds. Then, $\lambda_{r}\left|\partial_{l} B_{0}\left(l_{L}\right) \kappa_{L}\right| \gg$ $\left|\lambda-\lambda_{r}\right| B_{0}\left(l_{L}\right)$, and we can neglect $\left(\lambda-\lambda_{r}\right) B_{0}\left(l_{L}\right)$ in the quantity under the square root in (152). The same argument can be applied to the second term containing $g_{\mathrm{rl}}$ in (144). Therefore, if (151) is satisfied, (144) simplifies to

$$
\begin{gathered}
\left(\frac{Z_{i}}{T_{i}}+\frac{1}{T_{e}}\right) \varphi^{(1)}=\frac{2 \pi}{e n_{i}} \int_{0}^{\infty} \mathrm{d} v \int_{B_{0, \max }^{-1}}^{B^{-1}} \mathrm{~d} \lambda \frac{v^{3} B_{0}}{\left|v_{\|}^{(0)}\right|} g_{0}^{\text {out }} \\
+\frac{2 \pi B_{0}}{e n_{i}} \int_{v_{\min }}^{\infty} \mathrm{d} v \frac{v^{2}}{\sqrt{1-\lambda_{r} B_{0}(l)}} \int_{-\infty}^{\infty} \mathrm{d} \lambda g_{\mathrm{rl}}
\end{gathered}
$$

and $\widehat{J_{\varphi}^{(1)}}$, defined in (131), can be simply replaced by $J_{\varphi, r}^{(1)}$, where

$$
J_{\varphi, r}^{(1)}=-\frac{2 Z_{i} e}{m_{i} v} \int_{l_{L}}^{l_{R}} \frac{\varphi^{(1)}}{\sqrt{1-\lambda_{r} B_{0}(l)}} \mathrm{d} l .
$$

When $\varphi_{0}^{\prime}$ satisfies (151), we can solve (138) analytically. Its solution vanishing at infinity is

$$
g_{\mathrm{r} l, n}=-\frac{S_{r, n}}{\partial_{\lambda} \omega_{\alpha, r} n^{2 / 3} \lambda_{r} \beta} \int_{0}^{\infty} \exp \left(i \frac{n^{1 / 3}}{\beta} \frac{\lambda-\lambda_{r}}{\lambda_{r}} z-\frac{1}{3} z^{3}\right) \mathrm{d} z,
$$

where we have defined

$$
\beta:=\left(\frac{\nu_{\lambda} \chi_{r}}{\partial_{\lambda} \omega_{\alpha, r} \lambda_{r}^{3}}\right)^{1 / 3} \ll 1,
$$

$S_{r, n}$ are the coefficients of the Fourier expansion of $S_{r}$,

$$
S_{r}(\psi, \alpha, v):=S\left(\psi, \alpha, v, \lambda_{r}(\psi, v)\right),
$$

and $S=S_{B}+S_{\varphi}$ has been defined in (115). Note that for $S_{r}$ to be well defined, it is essential that (163) be correct as the asymptotic expression of $J_{\varphi}^{(1)}$ near $\lambda=\lambda_{r}$, and this is only true as long as condition (151) is met. Then, the contribution of resonant particles to $(67)$ is

$$
\begin{aligned}
& Q_{i, \mathrm{sb}-\mathrm{p}}=-\frac{2 \pi^{2} m_{i}^{2} c \delta^{2}}{Z_{i} e} \sum_{n=-\infty}^{\infty} i n \int_{v_{\min }}^{\infty} \mathrm{d} v v^{3}\left(\frac{v^{2}}{2}+\frac{Z_{i} e \varphi_{0}}{m_{i}}\right) \int_{-\infty}^{\infty} \mathrm{d} \lambda J_{r,-n}^{(1)} g_{\mathrm{rl}, n} \\
& =-\frac{4 \pi^{2} m_{i}^{3} c^{2} \delta^{2}}{Z_{i}^{2} e^{2} \Psi_{t}^{\prime}} \sum_{n=1}^{\infty} \int_{v_{\min }}^{\infty} \mathrm{d} v v^{3}\left(\frac{v^{2}}{2}+\frac{Z_{i} e \varphi_{0}}{m_{i}}\right) \frac{n^{4 / 3}}{\partial_{\lambda} \omega_{\alpha, r} \tau_{b, r}^{(0)} \lambda_{r}} \Upsilon_{i} F_{i 0} \int_{-\infty}^{\infty} \mathrm{d} \lambda\left|J_{r, n}^{(1)}\right|^{2}\{
\end{aligned}
$$




$$
\left.\frac{1}{\beta} \int_{0}^{\infty} \cos \left(\frac{n^{1 / 3}}{\beta} \frac{\lambda-\lambda_{r}}{\lambda_{r}} z\right) \exp \left(-\frac{1}{3} z^{3}\right) \mathrm{d} z\right\}
$$

where we have defined

$$
J_{r}^{(1)}(\psi, \alpha, v)=J^{(1)}\left(\psi, \alpha, v, \lambda_{r}(\psi, v)\right)
$$

and

$$
J_{r}^{(1)}(\psi, \alpha, v)=\sum_{n=-\infty}^{\infty} J_{r, n}^{(1)}(\psi, v) e^{i n \alpha}
$$

Next, we prove that the right side of (167) has a non-zero limit when $\beta \rightarrow 0$. For this, we employ the identity

$$
\lim _{\beta \rightarrow 0} \frac{1}{\beta} \int_{0}^{\infty} e^{-z^{3} / 3} \cos \left(\frac{1}{\beta} x z\right) \mathrm{d} z=\pi \delta(x)
$$

and the property $\delta(a x)=|a|^{-1} \delta(x)$, where $\delta(\cdot)$ is the Dirac delta distribution and $a$ is a real number. Then, for $\beta \ll 1$, the asymptotically dominant term is

$$
Q_{i, \mathrm{sb}-\mathrm{p}}=-\frac{4 \pi^{3} m_{i}^{3} c^{2} \delta^{2}}{Z_{i}^{2} e^{2} \Psi_{t}^{\prime}} \sum_{n=1}^{\infty} n \int_{v_{\min }}^{\infty} \mathrm{d} v v^{3}\left(\frac{v^{2}}{2}+\frac{Z_{i} e \varphi_{0}}{m_{i}}\right) \frac{1}{\partial_{\lambda} \omega_{\alpha, r} \tau_{b, r}^{(0)}} \Upsilon_{i} F_{i 0}\left|J_{n}^{(1)}\right|^{2} .
$$

\subsection{Formula for the ion energy flux when $\nu_{i *} \ll \rho_{i *}$}

Since the layers studied in subsections 4.1 and 4.2 are small and, in general, they are located around different points of phase space, their contributions to transport are additive. This means that we can write, for $\nu_{i *} \ll \rho_{i *}$,

$$
Q_{i}=Q_{i, \sqrt{\nu}}+Q_{i, \mathrm{sb}-\mathrm{p}}
$$

where $Q_{i, \sqrt{\nu}}$ is given by (106) and $Q_{i, \mathrm{sb}-\mathrm{p}}$ is given by (139). The weight of each term in (172) is determined by the value of $v_{\min }$. Typically, the estimate $(141)$ will be supressed by a factor $\exp \left(-v_{\min }^{2} / v_{t i}^{2}\right)$. Recalling also the estimate (113), we deduce that the superbanana-plateau regime dominates over the $\sqrt{\nu}$ regime when

$$
\frac{v_{\min }}{v_{t i}} \ll \sqrt{\ln \left(\frac{\omega_{\alpha}}{\nu_{i i}}\right)} .
$$

Conversely, the $\sqrt{\nu}$ regime dominates over the superbanana-plateau regime when

$$
\frac{v_{\min }}{v_{t i}} \gg \sqrt{\ln \left(\frac{\omega_{\alpha}}{\nu_{i i}}\right)} \text {. }
$$

Finally, we note that the value of $v_{\min }$ is set by the size of $\varphi_{0}^{\prime}$, but also by the specific $\lambda$-dependence of $\overline{\partial_{\psi} B_{0}}\left(\psi, \lambda_{r 0}\right)$ (recall condition $(121)$ ). 


\section{Calculation of the radial electric field}

The radial electric field, determined by $\varphi_{0}^{\prime}$, is one of the quantities that are routinely computed in standard neoclassical calculations. It is found by imposing that the radial electric current vanish.

Let us denote by $\Gamma_{i}$ and $\Gamma_{e}$ the radial fluxes of ions and electrons. The radial electric field is obtained by imposing

$$
Z_{i} e \Gamma_{i}-e \Gamma_{e}=0
$$

To lowest order in a mass ratio expansion $\sqrt{m_{e} / m_{i}} \ll 1$ this is equivalent to the condition

$$
\Gamma_{i}=0 .
$$

The calculation of $\Gamma_{i}$ is completely analogous to that of $Q_{i}$. Hence, asymptotically, (176) amounts to the condition

$$
\Gamma_{i, \sqrt{\nu}}+\Gamma_{i, \mathrm{sb}-\mathrm{p}}=0
$$

where

$\Gamma_{i, \sqrt{\nu}}=-\frac{2 \pi^{2} m_{i} c \delta^{2}}{Z_{i} e} \sum_{n=-\infty}^{\infty} i n \int_{0}^{\infty} \mathrm{d} v v^{3} \int_{\lambda_{c}}^{\lambda_{K}} \mathrm{~d} \lambda \widehat{J^{(1)}}{ }_{-n} g_{\mathrm{bl}, n}$

and

$\Gamma_{i, \mathrm{sb}-\mathrm{p}}=-\frac{2 \pi^{2} m_{i} c \delta^{2}}{Z_{i} e} \sum_{n=-\infty}^{\infty} i n \int_{v_{\min }}^{\infty} \mathrm{d} v v^{3} \int_{-\infty}^{\infty} \mathrm{d} \lambda\left[\left(J_{B, r}^{(1)}\right)_{-n}+\left(J_{\varphi}^{(1)}\right)_{-n}\right] g_{\mathrm{rl}, n}$.

\section{Estimation of $\nu_{\delta *}$}

In Section 4 we have solved the drift-kinetic equation and computed $Q_{i}$ when $\nu_{i *} \ll \rho_{i *}$. But we have advanced in the Introduction that our results are not valid for arbitrarily small $\nu_{i *}$. There exists a value of the collisionality, that we call $\nu_{\delta *}$, below which equation (172) is expected to be incorrect because the approximation to the drift-kinetic equation in (64) is incorrect. Hence, it is more precise to say that our results in Section 4 are correct when $\nu_{\delta *} \ll \nu_{i *} \ll \rho_{i *}$. In this section we explain the reason for the existence of $\nu_{\delta *}$ and estimate its value.

The limitations of equation (64) for sufficiently small $\nu_{i *}$ are well understood by inspecting the drift-kinetic equation written with the parallel velocity $u$ and the magnetic moment $\mu$ as independent coordinates. The drift-kinetic equation in terms of these coordinates is calculated in [11] to second order in a $\rho_{i *}$ expansion. If we denote by $\check{F}_{i}(\mathbf{R}, u, \mu)$ the distribution function expressed in coordinates $\{\mathbf{R}, u, \mu, \gamma\}$, and by $\check{F}_{i 1}(\mathbf{R}, u, \mu) \sim \delta \check{F}_{i}$ the deviation of $\check{F}_{i}(\mathbf{R}, u, \mu)$ from a Maxwellian distribution, we can check that the drift-kinetic equation in [11] contains a term of the form

$$
u \boldsymbol{\kappa} \cdot\left(\mathbf{v}_{\nabla B, i}+\mathbf{v}_{E, 0}\right) \partial_{u} \check{F}_{i 1} .
$$


Only the piece

$$
u\left[\boldsymbol{\kappa} \cdot\left(\mathbf{v}_{\nabla B, i}+\mathbf{v}_{E, 0}\right)\right]^{(0)} \partial_{u} \check{F}_{i 1} \sim \rho_{i *} \frac{v_{t i}}{L_{0}} \delta \check{F}_{i},
$$

corresponding to the omnigeneous magnetic field $\mathbf{B}_{0}$, enters (64). The effect of higherorder terms like

$$
u\left[\boldsymbol{\kappa} \cdot\left(\mathbf{v}_{\nabla B, i}+\mathbf{v}_{E, 0}\right)\right]^{(1)} \partial_{u} \check{F}_{i 1}
$$

has not been included. In Section 4 we learnt that transport is dominated by two collisional layers when $\nu_{i *} \ll \rho_{i *}$. In these layers, derivatives with respect to $u$ (or, equivalently, with respect to $\lambda$ ) are large, and they grow as $\nu_{i *}$ decreases. Let us denote by $\Delta_{u}$ the width of the layer in the coordinate $u$. The term (182) becomes comparable with the pitch-angle scattering piece of the collision operator when

$$
\frac{\delta \rho_{i *} v_{t i}}{L_{0}\left(\Delta_{u} / v_{t i}\right)} \sim \frac{\nu_{i i}}{\left(\Delta_{u} / v_{t i}\right)^{2}}
$$

If the stellarator is in the $\sqrt{\nu}$ regime, the boundary layer has a width $\Delta_{u}^{\sqrt{\nu}} / v_{t i} \sim \sqrt{\nu_{i *} / \rho_{i *}}$ and we get the estimation [31]

$$
\nu_{\delta *} \sim \delta^{2} \rho_{i *} .
$$

If the stellarator is in the superbanana-plateau regime, the size of the boundary layer is $\Delta_{u}^{\mathrm{sb}-\mathrm{p}} / v_{t i} \sim\left(\nu_{i *} / \rho_{i *}\right)^{1 / 3}$ and we get [32]

$$
\nu_{\delta *} \sim \delta^{3 / 2} \rho_{i *}
$$

When $\nu_{i *} \lesssim \nu_{\delta *}$, effects like those described in [33] must be taken into account. We leave this for future work.

\section{Conclusions}

Omnigeneity is the property of stellarators that have been perfectly optimized regarding neoclassical transport. It has been argued in [14] and in the Introduction of the present paper that, in practice, deviations from omnigeneity have a non-negligible effect on the neoclassical fluxes. It is natural to expect that this effect will be larger at low collisionality $\nu_{i *}$.

The $1 / \nu$ regime in stellarators close to omnigeneity is studied in [14]; this regime is defined by $\rho_{i *} \ll \nu_{i *} \ll 1$. In the core of hot stellarator plasmas, even lower collisionality regimes are relevant. The subject of this paper has been the study of the parameter range $\nu_{\delta *} \lesssim \nu_{i *} \lesssim \rho_{i *}$, with the restriction (39) for the perturbations of the omnigeneous configuration (i.e. the gradients of the perturbations have to be small).

When $\nu_{i *} \lesssim \rho_{i *}$, the components of the drifts tangential to the flux surface have to be retained. For a generic stellarator in this collisionality regime, the drift-kinetic equation becomes radially non-local. Transport in a stellarator close to omnigeneity conserves radial locality. The appropriate radially local drift-kinetic equation to solve for the dominant non-omnigeneous piece of the distribution function has been derived in Section 3. In Section 4 the equation has been solved and an explicit formula for the 
ion energy flux $Q_{i}$ has been provided in (172). The formula manifests, in particular, that when $\nu_{i *} \ll \rho_{i *}$ transport is determined by two small collisional layers. One of the layers is located around the boundary between trapped and passing particles and the other is located in the neighborhood of the phase-space points where the precession frequency (which is caused by the tangential drifts) vanishes. The former corresponds to the $\sqrt{\nu}$ regime and the latter to the superbanana-plateau regime. In addition, we have shown that the neoclassical fluxes scale with the square of the size of the deviation from omnigeneity.

In Section 4 we have also discussed the quasineutrality equation, employed to find the electric field tangent to the flux surface. We have proven that the superbananaplateau layer needs to be worked out in order to calculate the tangent electric field. The careful analysis of the quasineutrality equation showed that the specific form of the drift-kinetic equation in the superbanana-plateau regime depends on the size of the radial electric field. In Section 5 we have given the equation to determine the radial electric field.

Finally, in Section 6 we have explained why the results of Section 4 are not valid below a certain value of the collisionality, that we call $\nu_{\delta *}$ and that we have estimated. The treatment of the regime $\nu_{i *} \lesssim \nu_{\delta *}$ in stellarators close to omnigeneity is left for future work.

\section{Appendix A. Proof of relations (35) and (36)}

Starting from (15) and (16), let us first manipulate the radial components of the drifts. We employ that the radial $\nabla B$ drift can be conveniently rewritten with the help of the identity

$$
(\hat{\mathbf{b}} \times \nabla B) \cdot \nabla \psi=\frac{B}{\Psi_{t}^{\prime}}\left(-\partial_{\alpha} B+\partial_{\alpha} \mathbf{r} \cdot \hat{\mathbf{b}} \partial_{l} B\right),
$$

where we have used $\mathbf{B}=\Psi_{t}^{\prime} \nabla \psi \times \nabla \alpha, \partial_{l} \mathbf{r}=\hat{\mathbf{b}}$, and the relations

$$
\nabla \psi \times \nabla \alpha=\frac{1}{\sqrt{g}} \partial_{l} \mathbf{r} \quad \text { (and cyclic permutations of }\{\psi, \alpha, l\} \text { ), }
$$

with the volume element given by

$$
\sqrt{g}=\frac{\Psi_{t}^{\prime}}{B}
$$

Here, the position in euclidean coordinates is viewed as a function of the flux coordinates, $\mathbf{r}(\psi, \alpha, l)$.

In order to recast the radial curvature drift we use that $\boldsymbol{\kappa}=\hat{\mathbf{b}} \cdot \nabla \hat{\mathbf{b}}$ and that, trivially,

$\hat{\mathbf{b}} \cdot \nabla \hat{\mathbf{b}}=\left(\hat{\mathbf{b}} \cdot \nabla \hat{\mathbf{b}} \cdot \partial_{\psi} \mathbf{r}\right) \nabla \psi+\left(\hat{\mathbf{b}} \cdot \nabla \hat{\mathbf{b}} \cdot \partial_{\alpha} \mathbf{r}\right) \nabla \alpha+\left(\hat{\mathbf{b}} \cdot \nabla \hat{\mathbf{b}} \cdot \partial_{l} \mathbf{r}\right) \nabla l$.

The last term in the previous equation equals zero because $\partial_{l} \mathbf{r}=\hat{\mathbf{b}}$ and $\hat{\mathbf{b}} \cdot \nabla \hat{\mathbf{b}} \cdot \hat{\mathbf{b}} \equiv 0$. Then, it is easy to see that

$$
(\hat{\mathbf{b}} \times \boldsymbol{\kappa}) \cdot \nabla \psi=-\frac{B}{\Psi_{t}^{\prime}} \hat{\mathbf{b}} \cdot \nabla \hat{\mathbf{b}} \cdot \partial_{\alpha} \mathbf{r}
$$


Noting that $\hat{\mathbf{b}} \cdot \nabla \hat{\mathbf{b}}=\partial_{l} \hat{\mathbf{b}}$, integrating by parts in $l$ and using that $\hat{\mathbf{b}} \cdot \partial_{\alpha} \partial_{l} \mathbf{r}=\hat{\mathbf{b}} \cdot \partial_{\alpha} \hat{\mathbf{b}} \equiv 0$, we get

$$
(\hat{\mathbf{b}} \times \boldsymbol{\kappa}) \cdot \nabla \psi=-\frac{B}{\Psi_{t}^{\prime}} \partial_{l}\left(\hat{\mathbf{b}} \cdot \partial_{\alpha} \mathbf{r}\right) .
$$

Finally, the radial $E \times B$ drift will be rewritten by employing

$$
(\hat{\mathbf{b}} \times \nabla \varphi) \cdot \nabla \psi=\frac{B}{\Psi_{t}^{\prime}}\left(-\partial_{\alpha} \varphi+\partial_{\alpha} \mathbf{r} \cdot \hat{\mathbf{b}} \partial_{l} \varphi\right),
$$

which is obtained exactly in the same way as (A.1).

Recalling (15) and (16), and collecting the results (A.1), (A.6) and (A.7), we find

$$
\begin{aligned}
2 \int_{l_{b_{1}}}^{l_{b_{2}}} & \frac{1}{\left|v_{\|}\right|}\left(\mathbf{v}_{M, i}+\mathbf{v}_{E}\right) \cdot \nabla \psi \mathrm{d} l= \\
& \frac{2 m_{i} c}{Z_{i} e \Psi_{t}^{\prime}} \partial_{\alpha} \int_{l_{b_{1}}}^{l_{b_{2}}}\left|v_{\|}\right| \mathrm{d} l-\frac{2 m_{i} c}{Z_{i} e \Psi_{t}^{\prime}} \int_{l_{b_{1}}}^{l_{b_{2}}} \partial_{l}\left(\left|v_{\|}\right| \partial_{\alpha} \mathbf{r} \cdot \hat{\mathbf{b}}\right) \mathrm{d} l .
\end{aligned}
$$

Analogously, one can show that

$$
\begin{aligned}
2 \int_{l_{b_{1}}}^{l_{b_{2}}} & \frac{1}{\left|v_{\|}\right|}\left(\mathbf{v}_{M, i}+\mathbf{v}_{E}\right) \cdot \nabla \alpha \mathrm{d} l= \\
& \quad-\frac{2 m_{i} c}{Z_{i} e \Psi_{t}^{\prime}} \partial_{\psi} \int_{l_{b_{1}}}^{l_{b_{2}}}\left|v_{\|}\right| \mathrm{d} l+\frac{2 m_{i} c}{Z_{i} e \Psi_{t}^{\prime}} \int_{l_{b_{1}}}^{l_{b_{2}}} \partial_{l}\left(\left|v_{\|}\right| \partial_{\psi} \mathbf{r} \cdot \hat{\mathbf{b}}\right) \mathrm{d} l .
\end{aligned}
$$

The last term in both (A.8) and (A.9) vanishes because $v_{\|}$equals zero at $l_{b_{1}}$ and $l_{b_{2}}$. Finally, using definition (34), we obtain (35) and (36).

\section{Appendix B. Proof that the adiabatic response does not contribute to the energy flux}

The adiabatic response is contained in $F_{i 0}^{\mathcal{E}(0)}$, defined in (49). Its contribution to the energy flux (1) is given by

$Q_{i, \text { ad }}=4 \pi V^{\prime}(\psi)\left\langle\int_{Z_{i} e \varphi / m_{i}}^{\infty} \mathrm{d} \mathcal{E} \int_{0}^{B^{-1}\left(\mathcal{E}-Z_{i} e \varphi / m_{i}\right)} \mathrm{d} \mu \frac{B}{\left|v_{\|}\right|} m_{i} \mathcal{E} \mathbf{v}_{d, i} \cdot \nabla \psi F_{i 0}^{\mathcal{E}(0)}\right\rangle_{\psi}$,

with $\mathbf{v}_{d, i}=\mathbf{v}_{M, i}+\mathbf{v}_{E}$ (see definitions (15) and (16)). In (B.1) we have used that

$$
\int f \mathrm{~d}^{2} S=V^{\prime}(\psi)\langle f|\nabla \psi|\rangle_{\psi}
$$

where the flux surface average operation and $V^{\prime}(\psi)$ have been defined in (23) and (24).

A direct check shows that

$$
\mathbf{v}_{d, i} \cdot \nabla \psi=\frac{v_{\|}}{\Omega_{i}} \nabla \cdot\left(v_{\|} \hat{\mathbf{b}} \times \nabla \psi\right) .
$$


Then,

$$
\begin{gathered}
Q_{i, \text { ad }}=\frac{4 \pi m_{i}^{2} c}{Z_{i} e} V^{\prime}(\psi)\left\langle\int_{Z_{i} e \varphi / m_{i}}^{\infty} \mathrm{d} \mathcal{E} \int_{0}^{B^{-1}\left(\mathcal{E}-Z_{i} e \varphi / m_{i}\right)} \mathrm{d} \mu \nabla \cdot\left(\mathcal{E} F_{i 0}^{\mathcal{E}(0)}\left|v_{\|}\right| \hat{\mathbf{b}} \times \nabla \psi\right)\right\rangle_{\psi} \\
=\frac{4 \pi m_{i}^{2} c}{Z_{i} e} V^{\prime}(\psi)\left\langle\nabla \cdot \int_{Z_{i} e \varphi / m_{i}}^{\infty} \mathrm{d} \mathcal{E} \int_{0}^{B^{-1}\left(\mathcal{E}-Z_{i} e \varphi / m_{i}\right)} \mathrm{d} \mu\left(\mathcal{E} F_{i 0}^{\mathcal{E}(0)}\left|v_{\|}\right| \hat{\mathbf{b}} \times \nabla \psi\right)\right\rangle_{\psi}
\end{gathered}
$$

where we have used that $F_{i 0}^{\mathcal{E}(0)}$ is a flux function, and in the second equality we have employed that the integrand vanishes when $\mu=B^{-1}\left(\mathcal{E}-Z_{i} e \varphi / m_{i}\right)$ and when $\mathcal{E}=Z_{i} e \varphi / m_{i}$. Finally, recalling the identity

$$
\langle\nabla \cdot \mathbf{A}\rangle=\frac{1}{V^{\prime}(\psi)} \partial_{\psi}\left(V^{\prime}(\psi)\langle\mathbf{A} \cdot \nabla \psi\rangle_{\psi}\right)
$$

for any vector field $\mathbf{A}$ and applying it to (B.4) we deduce that $Q_{i \text {,ad }}$ vanishes.

\section{Appendix C. Asymptotic expansion of $\partial_{\psi} J^{(0)}$ near the boundary between trapped and passing particles}

We show that

$$
\partial_{\psi} J^{(0)}=-\int_{l_{b_{10}}}^{l_{b_{20}}} \frac{\lambda v \partial_{\psi} B_{0}+2 Z_{i} e /\left(m_{i} v\right) \partial_{\psi} \varphi_{0}}{\sqrt{1-\lambda B_{0}}} \mathrm{~d} l
$$

has the form (86) for small $\lambda-\lambda_{c}>0$ by, first, using the trivial identity

$$
\begin{aligned}
\partial_{\psi} J^{(0)} & =-\sum_{k=1}^{2} \int_{l_{b_{10}}}^{l_{b_{20}}} \frac{\lambda_{c} v \partial_{\psi} B_{0}\left(l_{M, k}\right)+2 Z_{i} e /\left(m_{i} v\right) \partial_{\psi} \varphi_{0}}{\sqrt{\left(\lambda_{c} / 2\right)\left|\partial_{l}^{2} B_{0}\left(l_{M, k}\right)\right|\left(l-l_{M, k}\right)^{2}-B_{0}\left(l_{M, k}\right)\left(\lambda-\lambda_{c}\right)}} \mathrm{d} l \\
& -\int_{l_{b_{10}}}^{l_{b_{20}}}\left(\frac{\lambda v \partial_{\psi} B_{0}(l)+2 Z_{i} e /\left(m_{i} v\right) \partial_{\psi} \varphi_{0}}{\sqrt{1-\lambda B_{0}(l)}}\right. \\
& \left.-\sum_{k=1}^{2} \frac{\lambda_{c} v \partial_{\psi} B_{0}\left(l_{M, k}\right)+2 Z_{i} e /\left(m_{i} v\right) \partial_{\psi} \varphi_{0}}{\sqrt{\left(\lambda_{c} / 2\right)\left|\partial_{l}^{2} B_{0}\left(l_{M, k}\right)\right|\left(l-l_{M, k}\right)^{2}-B_{0}\left(l_{M, k}\right)\left(\lambda-\lambda_{c}\right)}}\right) \mathrm{d} l,
\end{aligned}
$$

which is well defined for sufficiently small $\lambda-\lambda_{c}$. Here, we have only displayed the dependence of $B_{0}$ on $l$. The values $l_{M, k}$, for $k=1,2$, locate two consecutive absolute maxima of $B_{0}$ when moving along the field line; in particular, $B_{0}\left(l_{M, k}\right)=B_{0, \max }$ for $k=1,2$. The second integral on the right side of (C.2) is finite when $\lambda \rightarrow \lambda_{c}$, and hence it contributes to $a_{2}$ and higher-order terms in (86). The first integral on the right side of (C.2) can be computed analytically; namely,

$$
\begin{aligned}
& -\sum_{k=1}^{2} \int_{l_{b_{10}}}^{l_{b_{20}}} \frac{\lambda_{c} v \partial_{\psi} B_{0}\left(l_{M, k}\right)+2 Z_{i} e /\left(m_{i} v\right) \partial_{\psi} \varphi_{0}}{\sqrt{\left(\lambda_{c} / 2\right)\left|\partial_{l}^{2} B_{0}\left(l_{M, k}\right)\right|\left(l-l_{M, k}\right)^{2}-B_{0}\left(l_{M, k}\right)\left(\lambda-\lambda_{c}\right)}} \mathrm{d} l= \\
& -\sum_{k=1}^{2} \frac{\lambda_{c} v \partial_{\psi} B_{0}\left(l_{M, k}\right)+2 Z_{i} e /\left(m_{i} v\right) \partial_{\psi} \varphi_{0}}{\sqrt{\left(\lambda_{c} / 2\right)\left|\partial_{l}^{2} B_{0}\left(l_{M, k}\right)\right|}}
\end{aligned}
$$




$$
\times\left[\ln \left(|x|+\sqrt{x^{2}-\frac{2 B_{0}\left(l_{M, k}\right)}{\lambda_{c}\left|\partial_{l}^{2} B_{0}\left(l_{M, k}\right)\right|}\left(\lambda-\lambda_{c}\right)}\right)\right]_{x=l_{b_{10}}-l_{M, k}}^{x=l_{b_{20}}-l_{M, k}} .
$$

For small $\lambda-\lambda_{c}$,

$$
l_{b_{10}}-l_{M, 1}=\sqrt{\frac{2 B_{0}\left(l_{M, k}\right)\left(\lambda-\lambda_{c}\right)}{\lambda_{c}\left|\partial_{l}^{2} B_{0}\left(l_{M, k}\right)\right|}}+\ldots
$$

and

$$
l_{b_{20}}-l_{M, 2}=-\sqrt{\frac{2 B_{0}\left(l_{M, k}\right)\left(\lambda-\lambda_{c}\right)}{\lambda_{c}\left|\partial_{l}^{2} B_{0}\left(l_{M, k}\right)\right|}}+\ldots,
$$

whereas $l_{b_{20}}-l_{M, 1}=O\left(L_{0}\right)$ and $l_{b_{10}}-l_{M, 2}=O\left(L_{0}\right)$. Using these results in (C.3), it is straightforward to deduce that

$$
\begin{aligned}
& -\sum_{k=1}^{2} \int_{l_{b_{10}}}^{l_{b_{20}}} \frac{\lambda_{c} v \partial_{\psi} B_{0}\left(l_{M, k}\right)+2 Z_{i} e /\left(m_{i} v\right) \partial_{\psi} \varphi_{0}}{\sqrt{\left(\lambda_{c} / 2\right)\left|\partial_{l}^{2} B_{0}\left(l_{M, k}\right)\right|\left(l-l_{M, k}\right)^{2}-B_{0}\left(l_{M, k}\right)\left(\lambda-\lambda_{c}\right)}} \mathrm{d} l= \\
& \sqrt{\frac{1}{2 \lambda_{c}}} \sum_{k=1}^{2} \frac{\lambda_{c} v \partial_{\psi} B_{0}\left(l_{M, k}\right)+2 Z_{i} e /\left(m_{i} v\right) \partial_{\psi} \varphi_{0}}{\sqrt{\left|\partial_{l}^{2} B_{0}\left(l_{M, k}\right)\right|}} \ln \left(B_{0, \max }\left(\lambda-\lambda_{c}\right)\right)+O\left(v_{t i} L_{0} / \psi\right),
\end{aligned}
$$

from which equation (87) follows.

\section{Appendix D. Analysis of equation (101) in a neighborhood of $\lambda=\lambda_{c}$}

In this appendix we use the variable $x=\lambda-\lambda_{c}$ and rewrite (101) as

$$
\partial_{x}^{2} g_{n}+i n \frac{a_{1}}{\nu_{\lambda} \xi} \ln \left(\tilde{a}_{2} x\right) g_{n}=-\partial_{x}^{2} \underline{g_{0, n}},
$$

where $g_{n}(x)=g_{\mathrm{bl}, n}\left(\lambda_{c}+x\right)$ and $g_{0, n}(x)=g_{0, n}\left(\lambda_{c}+x\right)$. The equations (101) for $n \neq 0$ (recall that $g_{0}(x)$ and $g_{0,0}(x)$ vanish) have an irregular singular point [34] at $x=0$.

The standard methods do not work when applied to the homogeneous equation

$$
\partial_{x}^{2} g_{n}+i n \frac{a_{1}}{\nu_{\lambda} \xi} \ln \left(\tilde{a}_{2} x\right) g_{n}=0
$$

near $x=0$. However, one can check that the ansatz

$$
g_{n}=\sum_{m, p=0}^{\infty} A_{m, p} x^{2 p+m}(\ln x)^{p}
$$

is consistent, in the sense that by substitution in (D.2) one can find recurrence relations that determine all the coefficients $A_{m, p}$ except two of them. The free coefficients can be taken to be $A_{0,0}$ and $A_{1,0}$. In order to show this, it is advisable to start by writing the equation provided by terms in (D.2) that are proportional to $\ln x$ and the equation corresponding to terms proportional to $x \ln x$.

Hence, there exist two linearly independent solutions of (D.2) that are finite at $x=0$. 
It is easy to realize that the source term on the right side of (D.1) does not make $g_{n}$ diverge at $x=0$. First, note that $g_{0, n}$ is finite for any value of $x$. If one takes $g_{n}=-\underline{g_{0, n}}+f_{n},\left(\right.$ D.1) gives the following equation for $f_{n}$ :

$$
\partial_{x}^{2} f_{n}+i n \frac{a_{1}}{\nu_{\lambda} \xi} \ln \left(\tilde{a}_{2} x\right) f_{n}=\frac{i n}{\nu_{\lambda} \xi}\left(c_{1, n} \ln \left(\tilde{a}_{2} x\right)+\tilde{c}_{2, n}\right) \Upsilon_{i} F_{i 0},
$$

where $c_{1}$ and $\tilde{c}_{2}$ have been defined in (92) and (95). Since the indefinite integrals of $\ln x$ are finite everywhere, the source term on the right side of (D.4) does not introduce singularities in $f_{n}$ and we conclude that $g_{n}$ is finite for any value of $x$; in particular, it is finite at $x=0$.

\section{Acknowledgments}

This work has been carried out within the framework of the EUROfusion Consortium and has received funding from the Euratom research and training programme 20142018 under grant agreement No 633053. The views and opinions expressed herein do not necessarily reflect those of the European Commission. This research was supported in part by grants ENE2012-30832 and ENE2015-70142-P, Ministerio de Economía y Competitividad, Spain.

\section{References}

[1] Helander P, Beidler C D, Bird T M, Drevlak M, Feng Y, Hatzky R, Jenko F, Kleiber R, Proll J H E, Turkin Y and Xanthopoulos P 2012 Plasma Phys. Control. Fusion 54124009

[2] Cary J R and Shasharina S G 1997 Phys. Rev. Lett. 78674

[3] Cary J R and Shasharina S G 1997 Phys. Plasmas 43323

[4] Parra F I, Calvo I, Helander P and Landreman M 2015 Nucl. Fusion 55033005

[5] Landreman M and Catto P J 2012 Phys. Plasmas 19056103

[6] Hazeltine R D 1973 Plasma Phys. 1577

[7] Boozer A H 1983 Phys. Fluids 26496

[8] Nührenberg J and Zille R 1988 Phys. Lett. A 129113

[9] Connor J W, Fukuda T, Garbet X, Gormezano C, Mukhovatov V, Wakatani M, the ITB Database Groupa and the ITPA Topical Group on Transport and Internal Barrier Physics 2004 Nucl. Fusion 44 R1

[10] Garren D A and Boozer A H 1991 Phys. Fluids B 32822

[11] Calvo I, Parra F I, Velasco J L and Alonso J A 2013 Plasma Phys. Control. Fusion 55125014

[12] Calvo I, Parra F I, Alonso J A and Velasco J L 2014 Plasma Phys. Control. Fusion 56094003

[13] Calvo I, Parra F I, Velasco J L and Alonso J A 2015 Plasma Phys. Control. Fusion 57014014

[14] Parra F I, Calvo I, Velasco J L and Alonso J A "Neoclassical fluxes in the $1 / \nu$ regime of stellarators close to omnigeneity", in preparation

[15] Beidler C D et al 2011 Nucl. Fusion 51076001

[16] Logan N C, Park J K, Kim K, Wang Z and Berkery J W 2013 Phys. Plasmas 20122507

[17] Matsuoka S, Satake S, Kanno R and Sugama H 2015 Phys. Plasmas 22072511

[18] Dinklage A, Yokoyama M, Tanaka K, Velasco J L, López-Bruna D, Beidler C D, Satake S, Ascasíbar E, Arévalo J, Baldzuhn J, Feng Y, Gates D, Geiger J, Ida K, Jakubowski M, LópezFraguas A, Maassberg H, Miyazawa J, Morisaki T, Murakami S, Pablant N, Kobayashi S, Seki R, Suzuki C, Suzuki Y, Turkin Y, Wakasa A, Wolf R, Yamada H, Yoshinuma M, LHD Exp. Group, TJ-II Team, and W7-AS Team 2011 Nucl. Fusion 53063022 
[19] Galeev A A and Sagdeev R Z 1979 "Theory of neoclassical diffusion" Reviews of Plasma Physics, vol 7, p 257 ed Leontovich M A (New York: Consultants Bureau)

[20] Ho D D M and Kulsrud R M 1987 Phys. Fluids 30442

[21] Shaing K C 2015 J. Plasma Physics 81905810203

[22] García-Regaña J M, Kleiber R, Beidler C D, Turkin Y, Maassberg H and Helander P 2013 Plasma Phys. Control. Fusion $\mathbf{5 5} 074008$

[23] Pedrosa M A, Alonso J A, García-Regaña J M, Hidalgo C, Velasco J L, Calvo I, Kleiber R, Silva C and Helander P 2015 Nucl. Fusion 55052001

[24] Alonso J A, Velasco J L, Calvo I, Estrada T, Fontdecaba J M, García-Regaña J M, Geiger J, Landreman M, McCarthy K, Medina F, van Milligen B Ph, Ochando M A, Parra F I, the TJ-II team and the W7-X team 2016 Plasma Phys. Control. Fusion 58074009

[25] Parra F I and Catto P J 2008 Plasma Phys. Control. Fusion 50065014

[26] Parra F I and Calvo I 2011 Plasma Phys. Control. Fusion 53045001

[27] Catto P J 1978 Plasma Phys. 20719

[28] Helander P and Sigmar D J 2002 Collisional Transport in Magnetized Plasmas (Cambridge Monographs on Plasma Physics) ed Haines M G et al (Cambridge, UK: Cambridge University Press)

[29] L. M. Kovrizhnykh 1984 Nucl. Fusion 24851

[30] Parra F I, Calvo I, Velasco J L and Alonso J A "The second adiabatic invariant in stellarators close to omnigeneity", in preparation

[31] Shaing K C, Sabbagh S A and Chu M S 2009 Plasma Phys. Control. Fusion 51035004

[32] Shaing K C, Sabbagh S A and Chu M S 2009 Plasma Phys. Control. Fusion 51055003

[33] Mynick H E 1983 Phys. Fluids 262609

[34] Bender C M and Orszag S A 1999 Advanced Mathematical Methods for Scientists and Engineers I: Asymptotic Methods and Perturbation Theory. Springer, Berlin.

[35] Shaing K C, Sabbagh S A, Chu M S 2010 Nucl. Fusion 50025022 